\title{
Dynamic programming for stochastic target problems and geometric flows
}

Received October 24, 2000 / final version received July 24, 2001

Published online November 27, 2001 - (c) Springer-Verlag \& EMS 2001

\begin{abstract}
Given a controlled stochastic process, the reachability set is the collection of all initial data from which the state process can be driven into a target set at a specified time. Differential properties of these sets are studied by the dynamic programming principle which is proved by the Jankov-von Neumann measurable selection theorem. This principle implies that the reachability sets satisfy a geometric partial differential equation, which is the analogue of the Hamilton-Jacobi-Bellman equation for this problem. By appropriately choosing the controlled process, this connection provides a stochastic representation for mean curvature type geometric flows. Another application is the super-replication problem in financial mathematics. Several applications in this direction are also discussed.
\end{abstract}

\section{Introduction}

The stochastic target problem is a non-classical optimal stochastic control problem in which the controller tries to steer a controlled stochastic process $Z_{t, z}^{v}$ into a given target $G \subset \mathbb{R}^{n}$ at time $T$, by appropriately choosing a control process $v$. The object of interest is the collection of all initial data, $Z_{t, z}^{v}(t)=z$, from which the controller can achieve her goal:

$$
V(t):=\left\{z \in \mathbb{R}^{n}: Z_{t, z}^{v}(T) \in G \text { for some admissible } v\right\} .
$$

We call $V(t)$ the reachability set. As in classical optimal control, if the state process is Markov, then the reachability sets satisfy a dynamic programming principle which states that for any stopping time $\theta \in[t, T]$,

$$
V(t)=\left\{z \in \mathbb{R}^{n}: Z_{t, z}^{v}(\theta) \in V(\theta) \text { for some admissible } v\right\} .
$$

After properly defining the problem in a general abstract setup, we prove this identity in Theorem 3.1 under natural assumptions on the state process and the admissible controls. The main technical tools of this proof are a measurable selection

H. Mete Soner: Koç University, Department of Mathematics, Rumelifener Yolu, Sariyer, 80910 Istanbul, Turkey, e-mail: msoner@ku.edu.tr. Partially supported by the National Science Foundation grant DMS-98-17525.

N. Touzi: CREST, 15 Bd Gabriel Peri, 92245 Malakoff, Paris, France, e-mail: touzi@ensae.fr

Mathematics Subject Classification (1991): 49J20, 60J60; 49L20, 35K55

Part of this work was done at Princeton University, Department of Operations Research and Financial Engineering. Authors would like to thank for the hospitality. 
Theorem of Jankov-von Neumann and the techniques developed in Bertsekas and Shreve [6].

In Sect. 4, we use (1.1) to derive a partial differential equation when the state process is a diffusion process

$$
d Z_{t, z}^{v}(s)=\mu\left(s, Z_{t, z}^{v}(s), v(s)\right) d s+\sigma\left(s, Z_{t, z}^{v}(s), v(s)\right) d W(s)
$$

where $W$ is a $n$-dimensional Brownian motion, and the control $v$ is a progressively measurable process valued in a compact subset $U$ of some Euclidean space. In this general setup, the reachability set is neither a graph nor an epigraph. Therefore the evolution of the reachability sets can only be described by the geometric quantities of its boundary. We use the machinery developed in $[4,8,13,25]$ to derive and study the corresponding dynamic programming equation

$$
\sup \left\{-\mathcal{L}^{v} u(t, z): v \in \mathcal{N}(t, z, D u(t, z))\right\}=0 \quad(t, z) \in(0, T) \times \mathbb{R}^{n},
$$

where for $v \in U$,

$$
\mathcal{L}^{v} u(t, z):=u_{t}(t, z)+\mu(t, z, v)^{*} \nabla u(t, z)+\frac{1}{2} \operatorname{trace}\left(\sigma \sigma^{*}(t, z, v) D^{2} u(t, z)\right),
$$

and for $p \in \mathbb{R}^{n}$, and $(t, z) \in S=[0, T] \times \mathbb{R}^{n}$

$$
\mathcal{N}(t, z, p):=\left\{v \in U: \sigma^{*}(t, z, v) p=0\right\} .
$$

Since we wish to hit a deterministic target $G$ with probability one, the diffusion process has to degenerate along certain directions and the kernel $\mathcal{N}$ captures this fact.

The main result of Sect. 4 is Theorem 4.1 which states that the characteristic function of the complement of the reachability set is a viscosity solution of a nonlinear parabolic partial differential equation. Note that although the characteristic function is discontinuous, it solves a second order partial differential equation. This is common in the weak-viscosity theory for geometric flows; [4, 8, 13, 25].

This connection between the stochastic target problems and the geometric flows also provides a stochastic representation for the mean curvature flow, which was unexpected to the authors. The mean curvature flow is a geometric initial value problem for a family of sets $\{\Gamma(t)\}_{t \geq 0}$. Given the initial set $\Gamma(0)$, the problem is to construct $\Gamma(t)$ in such a way that the normal velocity of $\Gamma(t)$ at any point is equal to its mean curvature at that point. A brief discussion of this flow is given in Sect. 5 and we also refer to $[4,8,13,25]$ and the references therein for more information. In the case of the (codimension one) mean curvature flow the connection is this: Let the controlled process be

$$
d Z_{t, z}^{v}(s)=\left[I_{n}-v(s) v^{*}(s)\right] d W(s)
$$

where $I_{n}$ is the $n \times n$ identity matrix, and the control $v$ is any adapted unit vector. This choice provides a stochastic representation for the mean curvature flow problem, as a target reachability problem in the reverse time. More precisely, the characteristic function of the reachability set of this stochastic target problem is 
a weak-viscosity solution of the mean curvature flow. To the best of our knowledge, this is the first representation formula of this type. Note that it is analogous to the connection between forward-backward stochastic differential equations and the semilinear partial differential equations [23,22].

In general, the dynamic programming equation (1.2) corresponds to a general nonlinear geometric flow. Indeed formally (1.2) implies that the boundary of $V(t)$ satisfies the following equation:

$$
\text { (1.3) } \mathcal{V}=\sup \left\{-\mu(t, z, v) \cdot \vec{n}-\frac{1}{2} \operatorname{trace}\left(\sigma \sigma^{*}(t, z, v) D \vec{n}\right): v \in \mathcal{N}(t, z, \vec{n})\right\},
$$

where $\mathcal{V}$ is the normal velocity of the reachability set at $z \in \partial V(t)$, and $\vec{n}$ is outward unit vector at $z \in \partial V(t)$. A brief discussion of the geometric flows, the definition of $\mathcal{V}$ in terms of the distance function is given in Sect. 5.

The stochastic target problem was originally motivated by applications in financial mathematics. The reachability set is closely related to the super-replication problem which has been extensively studied in the last decade; we refer the reader to Karatzas and Shreve [18] and the references therein. In this financial pricing problem, one is interested in finding the minimal initial capital which allows to hedge some given contingent claim by means of an admissible portfolio strategy. Here, the control is the portfolio, the controlled stochastic process is the spot stock prices and the value of our portfolio, and the target is the set of all stock prices and portfolio values such that the portfolio value dominates a nonlinear function of stock prices given by the contingent claim.

Initialy, convex duality is used to analyze the super-replication problem. In general, when this approach is avaliable, the dual problem turns out to be a standard stochastic control problem (see e.g. Fleming and Soner [15]) which can be solved via the classical Hamilton-Jacobi-Bellman equations, see Cvitanić, Pham and Touzi [11]. Then the dual formulation admits a classical dynamic programming principle which is equivalent to the above one. Of course this derivation of the dynamic programming principle requires the convex duality result.

However, to this date, there is no general convex duality approach which applies to the general problem. In particular, there are several examples in financial mathematics which have not yet been solved by convex duality. Two such examples are the large investor framework (Cvitanić and $\mathrm{Ma}$ [10]), and the problem of hedging under Gamma constraints (Soner and Touzi [26]).

In this paper, we propose to study the target problem directly by the above dynamic programming principle 3.1. This alternative approach provides a way of deriving the Hamilton-Jacobi-Bellman equation directly and it has already been successfully applied in [26], [27] and [30].

In Sect. 6, we discuss super-replication problems with a Gamma constraint, transaction costs, and general portfolio constraints. In all of these examples, the reachability set is an epigraph of a function which is equal to the minimal superreplication price, and (1.1) yields a Hamilton-Jacobi-Bellman equation for the minimal super-replication price.

The paper is organized as follows. Section 2 describes the general stochastic target problem. Dynamic programming principle is proved in Sect. 3. The corres- 
ponding dynamic programming equation (1.2) is stated in Sect. 4. We defer the rigorous proof of the connection between the target problem and this equation to Sect. 8. In that section, we prove that the characteristic function of the complement of the reachability set is a viscosity solution of (1.2). In the remaining sections we discuss the applications. In Sect. 5, after a brief introduction to mean curvature flow, we obtain a stochastic representation for the mean curvature flow in any codimension. In Sect. 6, we discuss several applications in financial mathematics. An extension of the target reachability problem to the stochastic viability problem (Aubin et al. [2,3]) is given in Sect. 7.

Sections on financial mathematics, Sect. 6 and Sect. 7, and the section on mean curvature flow, Sect. 5 are independent of each other.

\section{Abstract problem}

In this section, we formulate the stochastic target problem. We start with a brief summary of the notation that will be used throughout the paper.

\subsection{Notation}

Let $(\Omega, \mathcal{F}, P)$ be a complete probability space, $T>0$ a finite time horizon, and $\mathbb{F}=\{\mathcal{F}(t), 0 \leq t \leq T\}$ a filtration satisfying the usual assumptions. $\mathcal{T}$ is the set of all stopping times in $[0, T]$.

For $\theta \in \mathcal{T}, L_{n}^{p}(\theta)$ is the set of all $p$ integrable, $\mathbb{R}^{n}$-valued random variables which are measurable with respect to $\mathcal{F}(\theta)$. For $\theta=T$, we simply denote $L_{n}^{p}:=$ $L_{n}^{p}(T)$. We also introduce the set $\mathcal{S}$ of all pairs $(\theta, \xi) \in \mathcal{T} \times L_{n}^{2}$ such that $\xi \in L_{n}^{2}(\theta)$. By analogy, we introduce the set $S=[0, T] \times \mathbb{R}^{n}$ which can be viewed as the subset of deterministic elements of $\mathcal{S}$.

Let $\mathbb{H}_{n}^{0}$ be the set of all càd-làg processes $X:[0, T] \times \Omega \longrightarrow \mathbb{R}^{n}$ progressively measurable with respect to the filtration $\mathbb{F}$, and $\boldsymbol{I H}_{n}^{p}$ the subset of $\boldsymbol{I H}_{n}^{0}$ whose elements satisfy $E\left[\int_{0}^{T}\left|X_{t}\right|^{p} d t\right]<\infty$.

For a topological space $A, \mathcal{B}_{A}$ is the set of all Borel subsets of $A$.

$M^{*}$ is the transpose of the matrix $M$.

\subsection{Admissible controls}

The control set $U$ is a Borel subset of an Euclidean space and $\mathcal{U}$ is the set of all progressively measurable processes $v:[0, T] \times \Omega \longrightarrow U$.

Given $\nu_{1}$ and $\nu_{2}$ in $\mathcal{U}$ and a stopping time $\theta \in \mathcal{T}$, we define the $\theta$-concatenation of $\left(v_{1}, v_{2}\right)$ by:

$$
v_{1} \stackrel{\theta}{\oplus} v_{2}:=v_{1} \mathbf{1}_{\llbracket 0, \theta)}+v_{2} \mathbf{1}_{\llbracket \theta, T \rrbracket} .
$$

Finally the set of admissible controls is any Borel subset $\mathcal{A}$ of $\mathcal{U}$ which satisfies the following two conditions. 
A1. Stability under concatenation:

$$
\nu_{1} \stackrel{\theta}{\oplus} \nu_{2} \in \mathcal{A} \text { for all } v_{1}, \nu_{2} \in \mathcal{A} \text { and } \theta \in \mathcal{T}
$$

This condition is crucial for dynamic programming. It essentially states that the set of admissible controls has an additive structure.

The second assumption is a technical condition and in many instances it follows from the topological structure we impose on $\mathcal{A}$. In particular, the following assumption holds if the set $\mathcal{A}$ is a separable, metric space; see Lemma 2.1 below.

\section{A2. Stability under measurable selection:}

We assume that for any $\theta \in \mathcal{T}$ and any measurable map

$$
\phi:(\Omega, \mathcal{F}(\theta)) \longrightarrow\left(\mathcal{A}, \mathcal{B}_{\mathcal{A}}\right)
$$

there exists $v \in \mathcal{A}$ such that

$$
\phi=v \quad \text { on } \llbracket \theta, T \rrbracket \times \Omega, \text { Leb } \times P \text { almost everywhere. }
$$

The issue here is this. Given $\phi$ as above we may define a control process $v$ by:

$$
v(t, \omega):=(\phi(\omega))(t, \omega) \mathbf{1}_{\{t \geq \theta\}}(\omega)+\bar{v}(t, \omega) \mathbf{1}_{\{t<\theta\}}(\omega),
$$

for some $\bar{v} \in \mathcal{A}$. Assumption $\mathbf{A} 2$ is then needed in order to show that $v$ is progressively measurable.

A sufficient condition for $\mathbf{A} 2$ is the separability of $\mathcal{A}$ :

Lemma 2.1. Suppose that $\mathcal{A}$ is a separable metric space. Then, $\mathbf{A} 2$ holds.

Proof. We first prove that the result holds for simple functions, then we deduce the result by density.

1. First suppose that $\phi$ is a simple function, i.e.,

$$
\phi=\sum_{k=1}^{\infty} v_{k} \mathbf{1}_{B_{k}},
$$

for some $v_{k} \in \mathcal{A}$ and pairwise disjoint sets $B_{k} \in \mathcal{F}_{\theta}$ whose union is the whole set $\Omega$. Let $v$ be as in (2.1). For any $t \in[0, T]$ and a Borel set $A \in \mathcal{B}_{U}$, we need to show that the set

$$
O:=\{(s, \omega) \in[0, t] \times \Omega \mid v(s, \omega) \in A\}
$$

is $\operatorname{Leb}_{[0, t]} \otimes \mathcal{F}_{t}$-measurable for all $t \geq 0$. Indeed, $O=O^{*} \cup\left(\cup_{k} O_{k}\right)$ where

$$
O^{*}:=\{s<\theta\} \cap\{\bar{v}(s, \omega) \in A\},
$$

and, with $\bar{B}_{k}:=[0, t] \times B_{k}$,

$$
O_{k}:=\{s \geq \theta\} \cap \bar{B}_{k} \cap\left\{v_{k}(s, \omega) \in A\right\} .
$$


Since $\bar{v}$ is progressively measurable, $O^{*}$ is $\operatorname{Leb}_{[0, t]} \otimes \mathcal{F}_{t}-$ measurable. Also for each $k, B_{k} \in \mathcal{F}_{\theta}$ and the definition of the $\sigma$-algebra $\mathcal{F}_{\theta},\{\theta \leq t\} \cap \bar{B}_{k}$ is $\operatorname{Leb}_{[0, t]} \otimes$ $\mathcal{F}_{t}$-measurable. Hence $O$ is $\operatorname{Leb}_{[0, t]} \otimes \mathcal{F}_{t}-$ measurable.

2. Since $\mathcal{A}$ is separable, there exist a sequence of maps $\phi_{n}: \Omega \rightarrow \mathcal{A}$ which are simple functions as in Step 1, and $\lim _{n} \phi_{n}=\phi$ everywhere. Let $v_{n}$ be as in (2.1) with $\phi_{n}$. Then, by Step $1, v_{n}$ is $\mathbb{F}$-progressively measurable and morevoever $v_{n}$ converges to $v$ everywhere. Hence $v$ is $\boldsymbol{F}$-progressively measurable as well.

\subsection{State process}

The controlled state process is a mapping from $\mathcal{S} \times \mathcal{A}$ into a subset $\mathcal{Z}$ of $\boldsymbol{H}_{n}^{0}$ :

$$
(\theta, \xi, v) \in \mathcal{S} \times \mathcal{A} \longmapsto Z_{\theta, \xi}^{v} \in \mathcal{Z} \subset \mathbb{H}_{n}^{0} .
$$

We shall denote

$$
\mathcal{Z}_{T}:=\left\{Z_{\theta, \xi}^{v}(T):(\theta, \xi, \nu) \in \mathcal{S} \times \mathcal{A}\right\} \subset L_{n}^{0} .
$$

The state process is assumed to satisfy the following conditions.

Z1. Initial data: $Z_{\theta, \xi}^{v}=0$ on $\left.\llbracket 0, \theta\right)$ and $Z_{\theta, \xi}^{v}(\theta)=\xi$.

Z2. Consistency with deterministic initial data: for all $(t, z) \in S$,

$$
Z_{\theta, \xi}^{v}=Z_{t, z}^{v} \text { on the event }\{(\theta, \xi)=(t, z)\} .
$$

The controlled process $Z^{v}$ is defined up to null sets, as an equivalence class in $\mathbb{H}_{n}^{2}$. Since the event $\{(\theta, \xi)=(t, z)\}$ may have zero measure, the above statement needs clarification. Precisely, by $\mathbf{Z 2}$ we mean that

$$
E\left[f\left(Z_{\theta, \xi}^{v}(s)\right) \mid(\theta, \xi)=(t, z)\right]=E\left[f\left(Z_{t, z}^{v}(s)\right)\right]
$$

for any bounded Borel function $f$ and $s \geq t$.

Z3. Pathwise uniqueness: for all $\tau \in \mathcal{T}$ with $\theta \leq \tau$ a.s., we have

$$
Z_{\theta, \xi}^{v}=Z_{\tau, \zeta}^{v} \text { on } \llbracket \tau, T \rrbracket \text { where } \zeta:=Z_{\theta, \xi}^{v}(\tau) \text {. }
$$

Z4. Causality: if two admissible controls $v_{1}, v_{2}$ are equal between two stopping times $\tau \geq \theta$ in $\mathcal{T}$, (i.e., $v_{1}=v_{2}$ on $\left.\llbracket \theta, \tau \rrbracket\right)$ then,

$$
Z_{\theta, \xi}^{\nu_{1}}=Z_{\theta, \xi}^{\nu_{2}} \text { on } \llbracket \theta, \tau \rrbracket \text {. }
$$

Z5. Measurability: the map

$$
(t, z, v) \in S \times \mathcal{A} \longmapsto Z_{t, z}^{v}(T) \in \mathcal{Z}_{T}
$$

is Borel measurable. 


\subsection{The stochastic control problem}

The target set $G$ is a Borel subset of $\mathbb{R}^{n}$. Given an initial data $(t, z) \in S$, let

$$
\mathcal{G}(t, z):=\left\{v \in \mathcal{A}: Z_{t, z}^{v}(T) \in G \text { a.s. }\right\}
$$

be the set of admissible controls which can drive the state process $Z$ into the target $G$ at the terminal time $T$. Observe that $\mathcal{G}(t, z)$ may be empty, and the reachability set is the collection of all points at which it is nonempty:

$$
V(t):=\left\{z \in \mathbb{R}^{n}: \mathcal{G}(t, z) \neq \varnothing\right\} .
$$

\subsection{Typical state and control processes}

In all of our applications, the state process $Z_{t, z}^{v}$ is a jump-diffusion process; see Sect. 6.1 below. Such a process is driven by a Brownian motion $W$ and random measures $\left\{v^{j}\right\}_{j=1}^{J}$. In these applications, we take the filtration $\mathcal{F}$ to be the $P$ completion of the smallest $\sigma$ algebra generated by the Brownian motion and the random measures.

Also a typical choice for the set of admissible controls $\mathcal{A}$ is a closed subset of adapted processes in $L^{p}((0, T) \times \Omega$; Lebesque $\otimes P)$ with some $p \in[1, \infty)$ and the product $\sigma$-algebra of $\mathcal{B}_{[0, T]} \times \mathcal{F}$. In view of Lemma 2.1, we would like $\mathcal{A}$ to be separable.

Indeed, since the set of progressively measurable processes is a closed subset of $L^{p}((0, T) \times \Omega)$, the separability of $\mathcal{A}$ follows from the separability of $L^{p}$. According to classical results on separability (see for instance, Doob [12], page 92), any $L^{p}$ space is separable if the underlying $\sigma$-algebra is countably generated upto null sets. Therefore, this choice of $\mathcal{A}$ is separable, provided that $\mathcal{F}$ is countably generated. We claim that this is always the case. Indeed, as it is discussed in Sect. 6.1, random measures $v^{j}$ are determined by the jump times $\left\{T_{n}^{j}\right\}_{n=1, \ldots}$ and random variables $\left\{Y_{n}^{j}\right\}_{n=1, \ldots}$. So that $\mathcal{F}$ is the $P$ completion of $\mathcal{F}^{0}=\mathcal{F}^{W} \vee \mathcal{F}^{T} \vee \mathcal{F}^{Y}$, where $\mathcal{F}^{W}$ is generated by the Brownian motion $W$, and $\mathcal{F}^{T}, \mathcal{F}^{Y}$ are generated by the sequence of random variables $\left\{T_{n}^{j}\right\},\left\{Y_{n}^{j}\right\}$, respectively. Since the Brownian paths are continuous $\mathcal{F}^{W}$ is countably generated (see for instance [24] Example 4.2.1 in Sect. 1). Therefore, $\mathcal{F}^{0}$ is countably generated and by construction, $\mathcal{F}$ is countably generated upto null sets.

Hence, with the above choices, $\mathcal{A}$ is separable, and Assumption $\mathbf{A} 2$ follows from Lemma 2.1.

\section{Dynamic programming}

We first start by a measurable selection result which is the key step in the proof of dynamic programming principle. Set

$$
D:=\{(t, z) \in S: z \in V(t)\}=\{(t, z) \in S: \mathcal{G}(t, z) \neq \emptyset\} .
$$


Lemma 3.1. For any probability measure $\mu$ on $S$, there exists a Borel measurable function $\phi_{\mu}:\left(D, \mathcal{B}_{D}\right) \longrightarrow\left(\mathcal{A}, \mathcal{B}_{\mathcal{A}}\right)$ such that:

$$
\phi_{\mu}(t, z) \in \mathcal{G}(t, z) \text { for } \mu-\text { a.e. }(t, z) \in D \text {. }
$$

Proof. By assumption, $S$ and $\mathcal{A}$ are Borel spaces. Set

$$
B:=\{(t, z, v) \in S \times \mathcal{A}: v \in \mathcal{G}(t, z)\} .
$$

1. We claim that $B$ is a Borel subset of $S \times \mathcal{A}$. Indeed, in view of $\mathbf{Z 5}$, the map $(t, z, v) \in S \times \mathcal{A} \longmapsto Z_{t, z}^{v}(T) \in \mathcal{Z}_{T}$ is Borel measurable. Therefore, for any bounded continuous function $f$, the map $\Psi_{f}:(t, z, v) \in S \times \mathcal{A} \longmapsto$ $E\left[f\left(Z_{t, z}^{v}(T)\right)\right] \in \mathbb{R}$ is Borel.

If $G$ is a closed subset of $\mathbb{R}^{n}$, then there exists a sequence of continuous functions $f^{n}$ such that $f^{n}(x) \longrightarrow \mathbf{1}_{G}(x)$ for all $x \in \mathbb{R}^{n}, f^{n}=1$ on $G$, and $0<f^{n} \leq 1$ outside $G$. Then, the map $\Psi_{\mathbf{1}_{G}}$ is Borel as limit of the Borel maps $\Psi_{f^{n}}$.

Now, if $G$ is open, then $\Psi_{\mathbf{1}_{G}}=1-\Psi_{\mathbf{1}_{G^{c}}}$ and therefore $\Psi_{\mathbf{1}_{G}}$ is Borel by step 1 . Clearly, this property extends to a countable union $\cup_{n} G_{n}$ of open or closed disjoint subsets of $\mathbb{R}^{n}$ since $\mathbf{1}_{\cup_{n} G_{n}}=\sum_{n} \mathbf{1}_{G_{n}}$. Hence, the map $\Psi_{\mathbf{1}_{G}}$ is Borel measurable for any Borel subset $G$ of $\mathbb{R}^{n}$.

Since $B=\left\{(t, z, v) \in S \times \mathcal{A}: \Psi_{\mathbf{1}_{G}}(t, z, v) \leq 0\right\}, B$ is a Borel set.

2. Since any Borel set is also analytic (see [6] Proposition 7.36), $B$ is an analytic subset of $S \times \mathcal{A}$. We may now apply the Jankov-von Neumann Theorem (see Bertsekas and Shreve [6] Proposition 7.49), to deduce the existence of an analytically measurable function $\phi: D \longrightarrow \mathcal{A}$ such that $\operatorname{Gr}(\phi) \subset B$, i.e. $\phi(t, z)$ is an admissible control in $\mathcal{G}(t, z)$ for all $(t, z) \in D$.

3. In this step, we will construct a Borel measurable map $\phi_{\mu}$ which is equal to $\phi, \mu$ almost everywhere.

Let $P(S)$ be the set of all probability measures on $S$, and for $\mu \in P(S)$, let $\mathcal{B}_{S}(\mu)$ the completion of the Borel $\sigma$-algebra $\mathcal{B}_{S}$ under $\mu$. Then $\mathcal{U}_{S}:=\cap_{\mu \in P(S)} \mathcal{B}_{S}(\mu)$ is called the universal $\sigma$-algebra. In view of Corollary 7.42.1 in Bertsekas and Shreve [6], every analytic subset of $S$ is universally measurable. In particular, any analytic map $\phi$ is universally measurable. Since $\mathcal{U}_{S} \subset \mathcal{B}_{S}(\mu)$ for any $\mu \in P(S)$, it follows that $\phi$ is $\mathcal{B}_{S}(\mu)$-measurable. Then, the definition of $\mathcal{B}_{S}(\mu)$ implies that there exists a Borel measurable map $\phi_{\mu}$ which is equal to $\phi$ for $\mu$ almost every $(t, z) \in S$.

We are now in a position to prove the dynamic programming principle.

Theorem 3.1. Let $(t, z) \in S$, and $\theta \geq t$ be a stopping time in $\mathcal{T}$. Then,

$$
V(t)=\left\{z \in \mathbb{R}^{n}: Z_{t, z}^{v}(\theta) \in V(\theta) \text { a.s. for some } v \in \mathcal{A}\right\} \text {. }
$$

Proof. Set $W(t):=\left\{z \in \mathbb{R}^{n}: Z_{t, z}^{v}(\theta) \in V(\theta)\right.$ a.s. for some $\left.v \in \mathcal{A}\right\}$.

1. We first prove that $V(t) \subset W(t)$. Let $z$ be an arbitrary element of $V(t)$. Then, there exists $v \in \mathcal{A}$ such that $Z_{t, z}^{v}(T) \in G$ a.s.. The pathwise uniqueness property 
$\mathbf{Z 3}$ yields

$$
Z_{t, z}^{v}(T)=Z_{\theta, Z_{t, z}^{v}(\theta)}^{v}(T) \in G \text { a.s.. }
$$

Let $\mu=\mu_{\theta, t, z}$ be the pull-back of $P$ under the map $\left(\theta, Z_{t, z}^{v}(\theta)\right)$, as such $\mu$ is a Lebesgue measure on $[0, T] \times \mathbb{R}^{n}$. We need to show that for $\mu$-a.e. $\left(t^{\prime}, z^{\prime}\right)$, $z^{\prime} \in V\left(t^{\prime}\right)$

By $\mathbf{Z 2}$, for every $\left(t^{\prime}, z^{\prime}\right)$,

$$
P\left(Z_{t^{\prime}, z^{\prime}}^{v}(T) \in G\right)=P\left(Z_{\theta, Z_{t, z}^{v}(\theta)}^{v}(T) \in G \mid\left(\theta, Z_{t, z}^{v}(\theta)\right)=\left(t^{\prime}, z^{\prime}\right)\right) .
$$

Hence

$$
\begin{aligned}
\int P\left(Z_{t^{\prime}, z^{\prime}}^{v}(T) \in G\right) d \mu & =E\left(P\left(Z_{\theta, Z_{t, z}^{v}(\theta)}^{v}(T) \in G \mid\left(\theta, Z_{t, z}^{v}(\theta)\right)=\left(t^{\prime}, z^{\prime}\right)\right)\right) \\
& =P\left(Z_{\theta, Z_{t, z}^{v}(\theta)}^{v}(T) \in G\right) \\
& =P\left(Z_{t, z}^{v}(T) \in G\right)=1 .
\end{aligned}
$$

Hence for $\mu$-a.e. $\left(t^{\prime}, z^{\prime}\right), P\left(Z_{t^{\prime}, z^{\prime}}^{v}(T) \in G\right)=1$, and $z^{\prime} \in V\left(t^{\prime}\right)$. Therefore, $Z_{t, z}^{v}(\theta) \in V(\theta)$ a.s.

2. In this step, we prove the opposite inclusion $W(t) \subset V(t)$. Let $(t, z) \in W(t)$. Then,

$$
Z_{t, z}^{v}(\theta) \in V(\theta) \text { a.s. for some } v \in \mathcal{A} \text {. }
$$

Let $\mu$ be the probability measure on $S$ induced by $\left(\theta, Z_{t, z}^{v}(\theta)\right)$, and let $\phi_{\mu}$ be the Borel measurable map constructed in Lemma 3.1. In view of (3.2), $\left(\theta, Z_{t, z}^{v}(\theta)\right)$ $\in D$ a.s.. Then by Lemma 3.1 and $\mathbf{A 2}$, there exists $\nu_{1} \in \mathcal{A}$ such that for $\mu$-almost every $\left(t^{\prime}, z^{\prime}\right)$,

$$
Z_{t^{\prime}, z^{\prime}}^{v_{1}}(T)=Z_{t^{\prime}, z^{\prime}}^{\phi_{\mu}\left(z^{\prime} z^{\prime}\right)}(T) \in G \text { on the event set }\left\{\left(\theta, Z_{t, z}^{v}(\theta)\right)=\left(t^{\prime}, z^{\prime}\right)\right\} .
$$

Set $\hat{v}:=v \stackrel{\theta}{\oplus} v_{1}$. According to the stability by concatenation property $\mathbf{A 1}, \hat{v}$ is an admissible control in $\mathcal{A}$. We now compute that

$$
\begin{aligned}
Z_{t, z}^{\hat{v}}(T) & =Z_{\theta, Z_{t, z}^{\hat{v}}(\theta)}^{\hat{v}}(T) \quad \text { by } \mathbf{Z 3} \\
& =Z_{\theta, Z_{t, z}^{v}(\theta)}^{\hat{v}}(T) \quad \text { by } \mathbf{Z 4} \text { since } \hat{v}=v \text { on } \llbracket t, \theta \rrbracket \\
& =Z_{\theta, Z_{t, z}^{v}(\theta)}^{v_{1}}(T) \quad \text { by } \mathbf{Z 4} \text { since } \hat{v}=v_{1} \text { on } \llbracket \theta, T \rrbracket \\
& \in G \quad \text { by }(3.3) .
\end{aligned}
$$

Hence $\hat{v} \in \mathcal{G}(t, z)$ and $z \in V(t)$. 


\section{Dynamic programming equation}

In this section we state the corresponding dynamic programming equation when the state process is a diffusion.

In our earlier work, [26] [27], the dynamic programming property is used to study two problems in finance. In those two examples, the reachability set $V(t)$ is the epigraph of the value functions. In [26] and [27] this structure and the dynamic programming principle (3.1) is then used to derive a nonlinear partial differential equation for these functions.

In the general setup outlined in this paper, the reachability set $V(t)$ may not be an epigraph. Still, the dynamic programming principle (3.1) can be used to derive an equation for the boundary of the reachability set. Necessarily, this equation is a geometric flow equation as defined in Barles, Soner and Souganidis [4]. This connection between the stochastic target problems and the geometric flows is further discussed in our forthcoming papers [29] and [28]. In particular, in [29], we provide a stochastic representation formula for the weak solutions of the mean curvature flow as defined in Chen, Giga, Goto [8], Evans, Spruck [13] and Ambrosio, Soner [1]. To our knowledge, this is the first representation formula of this type. A brief discussion of this formula is given in the next section.

In this section, we simply state this "geometric dynamic programming equation" which is the analogue of the Bellman equation of a standard optimal control problem. We then discuss the properties of this equation and later in Sect. 8 we establish the connection between the target problem and this equation by proving that the complement of the characteristic function of the reachability set is a viscosity solution.

We assume that the state process $Z_{t, z}^{v}$ is a diffusion process solving

$$
d Z_{t, z}^{v}(s)=\mu\left(s, Z_{t, z}^{v}(s), v(s)\right) d s+\sigma\left(s, Z_{t, z}^{v}(s), v(s)\right) d W(s),
$$

where $W$ is a $n$-dimensional Brownian motion. We assume that $\mu$ and $\sigma$ are both bounded and satisfy the usual Lipschitz conditions, and that the control set $U$ is compact. The Dynkin operator associated with the controlled diffusion $Z^{v}$ will be denoted by:

$$
\mathcal{L}^{v} u(t, z):=u_{t}(t, z)+\mu(t, z, v)^{*} D u(t, z)+\frac{1}{2} \operatorname{trace}\left(\sigma \sigma^{*}(t, z, v) D^{2} u(t, z)\right) .
$$

Further, for $p \in \mathbb{R}^{n}$, and $(t, z) \in S=[0, T] \times \mathbb{R}^{n}$ let,

$$
\mathcal{N}(t, z, p):=\left\{v \in U: \sigma^{*}(t, z, v) p=0\right\} .
$$

Note that $\mathcal{N}(t, z, 0)=U$ for any $(t, z)$. In the rest of this section, we shall assume that the following standing assumption

$$
\mathcal{N}(t, z, p) \neq \varnothing \text { for all }(t, z, p) \in[0, T) \times \mathbb{R}^{n} \times \mathbb{R}^{n}
$$

holds.

The dynamic programming equation for this diffusion target problem is

$$
-u_{t}(t, z)+F\left(t, z, D u(t, z), D^{2} u(t, z)\right)=0,
$$


where, for $p \in \mathbb{R}^{n}, p \neq 0,(t, z) \in S$ and a symmetric matrix $A$,

$F(t, z, p, A):=\sup \left\{-\mu(t, z, \nu)^{*} p-\frac{1}{2} \operatorname{trace}\left(\sigma \sigma^{*}(t, z, \nu) A\right): v \in \mathcal{N}(t, z, p)\right\}$,

so that $-u_{t}(t, z)+F\left(t, z, D u(t, z), D^{2} u(t, z),\right)=\sup \left\{-\mathcal{L}^{v}(t, z): v \in \mathcal{N}(t, z\right.$, $D u(t, z))\}$.

The connection between the diffusion target problem and the above nonlinear equation is this. Let $u(t, z)$ be the characteristic function of the complement of the reachability set $V(t)$,

$$
u(t, z):=1-\mathbf{1}_{V(t)}(z):=\left\{\begin{array}{l}
0 \text { for } z \in V(t) \\
1 \text { for } z \notin V(t) .
\end{array}\right.
$$

We are ready to state the main theorem which establishes a connection between the stochastic target and the equation (4.1). This requires the following technical condition on the set valued function $\mathcal{N}(t, z, p)$ :

Assumption 4.1. (Continuity of $\mathcal{N}(t, z, p))$ For any $\left(t_{0}, z_{0}, p_{0}\right) \in S \times \mathbb{R}^{n}$ and $v_{0} \in \mathcal{N}\left(t_{0}, z_{0}, p_{0}\right)$, there exists a map $\hat{v}: S \times \mathbb{R}^{n} \longrightarrow U$ satisfying,

$$
\begin{aligned}
& \hat{v}\left(t_{0}, z_{0}, p_{0}\right)=v_{0}, \\
& \hat{v}(t, z, p) \in \mathcal{N}(t, z, p) \text { for all }(t, z, p) \in S \times \mathbb{R}^{n}, \\
& \hat{v} \text { is locally Lipschitz on }\{(t, z, p): p \neq 0\} .
\end{aligned}
$$

Theorem 4.1. Assume that $U$ is compact and $\mathcal{N}(t, z, p)$ satisfies the continuity Assumption 4.1. Further assume that $F$ is locally Lipschitz on $\{p \neq 0\}$. Then, $u$ is a discontinuous viscosity solution of (4.1).

We defer the proof of this theorem to Sect. 8. We refer to Chen, Giga and Goto (1991) for the discussion of the uniqueness issue related to the above non-linear PDE.

Recall that $u$ is a discontinuous viscosity supersolution (resp. subsolution) of (4.1), if the lower semicontinuous envelope $u_{*}$ (resp. the upper semicontinuous envelope $u^{*}$ ) of $u$ is a viscosity supersolution (resp. subsolution) of the equation $-\left(u_{*}\right)_{t}+F^{*}\left(t, z, D u_{*}, D^{2} u_{*}\right) \geq 0\left(\right.$ resp. $\left.-\left(u^{*}\right)_{t}+F_{*}\left(t, z, D u^{*}, D^{2} u^{*}\right) \leq 0\right)$.

As discussed in the introduction, this equation is the level set equation for the geometric equation (1.3). Thus the above problem implies that the reachability set satisfies the geometric equation. This property is discussed in detail in Sect. 5. In particular we will show that when the resulting geometric flow has a smooth solution then it is equal to $V(t)$.

Note that although $u$ is discontinuous, it solves a second order partial differential equation. In this connection, the definition of $\mathcal{N}(t, z, p)$ plays a crucial role which implies that the equation (4.1) is degenerate along the $D u$ direction. This essentially means that the dynamic programming equation (4.1) is a geometric equation only 
for the boundary of $V(t)$; see Barles, Soner, Souganidis [4]. Indeed, the nonlinearity $F$ has the following two important properties

$$
\begin{array}{rlrl}
F\left(t, z, c_{1} p, c_{1} A+c_{2} p p^{*}\right) & =c_{1} F(t, z, p, A) & & \forall c_{1}>0, c_{2} \in \mathbb{R}, \\
F(t, z, p, A+B) \leq F(t, z, p, A), & & \forall B \geq 0 .
\end{array}
$$

The second property means that (4.1) is elliptic, while the first implies that it geometric. Note that the geometric property implies that (4.1) is degenerate along the gradient direction which is the normal direction to the level sets of $u$.

In some examples, the reachability set $V(t)$ may be lower dimensional. In that case, $u^{*} \equiv 1$ and therefore $u$ is automatically a viscosity subsolution of (4.1). Interestingly, still the supersolution property of $u$ contains enough information; see [1] Sect. 3.

We conclude this section by the following remark, which illustrates the typical use of Assumption 4.1, and provides a lower bound for $V(t)$.

Remark 4.1. (i) Fix $(t, z)$, a small constant $\delta>0$ and $\hat{v}$ be as in Assumption 4.1. Construct processes $v$ and $Z_{z}^{v}$ so that for all $s \in[t, T]$,

$$
v(s)=\hat{v}\left(s, Z_{z}^{v}(s), Z_{z}^{v}(s)-z\right), \quad \text { whenever }\left|Z_{z}^{v}(s)-z\right| \geq \delta .
$$

Set $y(s):=Z_{z}^{v}(s)-z$ and apply Itô's rule to $|y(s)|^{2}$,

$$
d|y(s)|^{2}=\left[2 y(s)^{*} \mu(\cdots)+\operatorname{trace}\left(\sigma \sigma^{*}(\cdots)\right)\right] d s+2 y(s)^{*} \sigma(\cdots) d W(s),
$$

where $(\cdots)=(s, y(s), v(s))$. Since $v(s) \in \mathcal{N}\left(s, Z_{z}^{v}(s), y(s)\right)$ whenever $|y(s)| \geq \delta$, the stochastic term in the above equation is equal to zero. Hence, when $|y(s)| \geq \delta$,

$$
d|y(s)|^{2} \leq C(|y(s)|+1) d s,
$$

for some constant $C$, depending on the bounds on $\mu$ and $\sigma$. This proves that:

$$
\begin{aligned}
|y(s)|^{2} & \leq \delta^{2}+C \int_{t}^{s}(1+|y(r)|) \mathbf{1}_{|y(r)| \geq \delta} d r \\
& \leq \delta^{2}+C(s-t)+\frac{C}{\delta} \int_{t}^{s}\left|y(r)^{2}\right| d r .
\end{aligned}
$$

We now use Gronwall's Lemma to see that

$$
|y(s)|^{2} \leq h(\delta):=C \delta(1+\delta)+\left(\delta+\delta^{2}\right) e^{C \delta} \text { for }(s-t) \leq \delta^{2}
$$

Since $\delta$ is arbitrary, this proves that, with a convenient choice of the control $v$, the distance between $Z_{z}^{v}(s)$ and $z$ can be controlled in small time.

(ii) Now suppose that the target $G$ has non-empty interior. Then from the latter observation, for small $\delta>0$, let $G_{\delta}$ be a subset of $G$, with non-empty interior, and satisfying $\operatorname{dist}\left(\partial G_{\delta}, \partial G\right)>h(\delta)$. Then, clearly $G_{\delta}$ is a lower bound for $V\left(T-\delta^{2}\right)$ in the sense that $G_{\delta} \subset V\left(T-\delta^{2}\right)$. 


\section{Application to mean curvature flow}

An important example of a parabolic geometric flow is the mean curvature flow. In this section, we will show that our results provide a stochastic representation for the solutions of this flow. There is a large literature on mean curvature flow. We refer to [19], [20], and [21] for the analysis of smooth solutions and their properties, and we refer to [8], [13] and [1] and the references therein for the weak-viscosity solutions of geometric flows. Further study of this connection is given in [28].

Smooth mean curvature flow is a family of smooth manifolds $\{\Gamma(t)\}_{t \geq 0}$ indexed by the time variable $t$. At any point $x \in \Gamma(t)$, the normal velocity $\mathcal{V}=\mathcal{V}(t, x)$ at that point is equal to a constant times the mean curvature $\mathcal{H}=\mathcal{H}(t, x)$ of the smooth set $\Gamma(t)$, i.e.,

$$
\mathcal{V}=c^{*} \mathcal{H}
$$

for some positive constant $c^{*}$. We may view this flow as a geometric initial value problem: given $\Gamma(0)$ find the time evolution $\Gamma(t)$ so that $(5.1)$ holds everywhere.

In this paper, we only consider the case when these manifolds are subsets of an Euclidean space $\mathbb{R}^{n}$. In this case, we may define all the relevant geometric quantities, such as $\mathcal{V}$ and $\mathcal{H}$, in terms of the distance function. Since the constant $c^{*}$ can be changed by appropriately scaling time, we will take $c^{*}=(n-k)$, where $k$ is codimension of the sets $\Gamma(t)$.

Let us start with the case when $\Gamma(t)$ is a smooth hypersurface enclosing an open set $\mathcal{O}(t)$ so that the signed distance function:

$$
\rho(t, x):= \begin{cases}\operatorname{distance}(x, \Gamma(t)), & \text { if } \quad x \in \mathcal{O}(t), \\ -\operatorname{distance}(x, \Gamma(t)), & \text { if } \quad x \notin \mathcal{O}(t)\end{cases}
$$

is smooth in some tubular neighborhood $\{(t, x):|\rho(t, x)| \leq \varepsilon\}$. Then, at $x \in \Gamma(t)$, the normal velocity and the mean curvature are given by

$$
\mathcal{V}(t, x)=\rho_{t}(t, x), \quad \mathcal{H}(t, x)=\frac{1}{n-1} \Delta \rho(t, x) .
$$

Since $c^{*}=(n-1)$, the signed distance function of a mean curvature flow $\{\Gamma(t)\}_{t \geq 0}$ satisfies

$$
\rho_{t}(t, x)=\Delta \rho(t, x), \quad \forall x \in \Gamma(t) .
$$

Note that $x \in \Gamma(t)$, if and only if $\rho(t, x)=0$. Hence the signed distance function $\rho$ satisfies the heat equation only on its zero level set. Away from its zero set, it does not satisfy the heat equation, however, it satisfies certain inequalities which are useful in the study of mean curvature flow. Indeed it is shown in [25] that

$$
\left[\rho_{t}-\Delta \rho\right] \rho \geq 0
$$

Since $\rho$ is not smooth everywhere, the above inequality has to be interpreted in the viscosity sense. 
Example 5.1. As a simple example consider the evolution of a sphere $\Gamma(0)=$ $\partial B_{R_{0}}:=\left\{x \in \mathbb{R}^{n}|| x \mid=R_{0}\right\}$. Since the mean curvature flow is invariant under rotation, we guess the solution $\Gamma(t)$ to be a sphere as well, i.e., $\Gamma(t)=\partial B_{R(t)}$. Then, the normal velocity and the mean curvature of $\Gamma(t)$ are easy to calculate,

$$
\mathcal{V}=\frac{d}{d t} R(t), \quad \mathcal{H}=-\frac{1}{R(t)} .
$$

Then, the equation (5.1) implies that

$$
\frac{d}{d t} R(t)=-\frac{(n-1)}{R(t)} \Rightarrow R(t)=\sqrt{R(0)^{2}-2(n-1) t}
$$

Note that $\Gamma(t)$ becomes empty for all $t>\sqrt{R(0)^{2} / 2(n-1)}$.

When $\Gamma(t)$ has codimension higher than one, we can not define a signed distance function and the distance function is not smooth on $\Gamma(t)$. However, the square distance function

$$
\eta(t, x):=\frac{1}{2}(\operatorname{distance}(x, \Gamma(t)))^{2}
$$

is as smooth as $\Gamma(t)$ in a tubular neigborhood of $\Gamma(t)$. Let $\overrightarrow{\mathcal{V}}(t, x), \overrightarrow{\mathcal{H}}(t, x)$ be, respectively, the normal velocity vector and the mean curvature vector. Then, $\overrightarrow{\mathcal{V}}=\mathcal{V} \vec{n}$, and $\overrightarrow{\mathcal{H}}=\mathcal{H} \vec{n}$, for some unit normal vector $\vec{n}$; see [1] for the definition of $\vec{H}$. Then, following a suggestion of DeGiorgi it is shown that (see [1])

$$
\overrightarrow{\mathcal{V}}(t, x)=D \eta_{t}(t, x), \quad \overrightarrow{\mathcal{H}}(t, x)=D \Delta \eta(t, x) .
$$

Therefore, if $\{\Gamma(t)\}_{t \geq 0}$ is a mean curvature, then

$$
D \eta_{t}(t, x)=D \Delta \eta(t, x), \quad \text { on }\{(t, x): \eta(t, x)=0\} .
$$

Planar mean curvature flow is also known as the curve shortening equation. In a series of papers, it is shown that solutions of the curve shortening equation remain smooth and shrink to a point in finite time [19-21]. However, in higher dimensions mean curvature flow do create singularities even if it starts smoothly. For that reason several weak-viscosity solutions have been introduced [8,13,25]. They have shown that the following partial differential equation can be used to characterize the viscosity solutions of the (codimension one) mean curvature flow.

$$
-u_{t}+F_{1}\left(D^{2} u, D u\right)=0
$$

where for $p \in \mathbb{R}^{n}, p \neq 0$, and a symmetric matrix $A$,

$$
F_{1}(A, p):=-\operatorname{trace}\left[A \Pi^{p}\right], \quad \Pi^{p}:=I_{n}-\left(p p^{*}\right) /|p|^{2} .
$$

Later a similar differential equation is obtained for higher codimension flows [1]. For flows with codimension $k$, the equation is

$$
-u_{t}+F_{k}\left(D^{2} u, D u\right)=0
$$


with

$$
F_{k}(A, p):=-\sum_{i=1}^{n-k} \lambda_{i}^{p}(A),
$$

where $\lambda_{1}^{p}(A) \leq \lambda_{2}^{p}(A) \leq \ldots \leq \lambda_{n-1}^{p}(A)$ are the eigenvalues of $\Pi^{p} A \Pi^{p}$ corresponding to eigenvectors which are orthogonal to $p$.

In the next two subsections we will obtain a stochastic representation of the Euclidean mean curvature flow. Although general codimension case can be handled directly, for the ease of presentation we will treat the codimension one case first and then describe the general codimension.

\subsection{Codimension one mean curvature flow}

Since for the mean curvature flow it is appropriate to assign an initial set, we reverse time and consider the following state dynamics

$$
d Z_{z}^{v}(s)=\sqrt{2}\left(I_{n}-v(s) v^{*}(s)\right) d W(s), \quad s>0,
$$

with initial data $Z_{z}^{v}(0)=z$. Control processes take values in $U=S^{n-1}=\{z \in$ $\left.\mathbb{R}^{n}:|z|=1\right\}$ and $I_{n}$ is the $n \times n$ identity matrix.

With the notation of the previous section, $\mathcal{N}(t, z, p)=\{p /|p|,-p /|p|\}$ for any $p \neq 0$ and therefore the continuity Assumption 4.1 is satisfied. Then the dynamic programming equation (4.1) (after changing the direction of time) takes the form

$$
\begin{aligned}
u_{t} & =-\sup \left\{-\Delta u+D^{2} u v \cdot v \mid v \in \mathcal{N}(t, z, D u)\right\} \\
& =\Delta u-\frac{D^{2} u(t, z) D u(t, z) \cdot D u(t, z)}{|D u(t, z)|^{2}}
\end{aligned}
$$

This is exactly the level set equation of the (codimension one) mean curvature flow. Note that the above equation is not defined when $D u(t, z)=0$. This problem is addressed in detail in the papers on weak-viscosity solutions of the mean curvature flow. See also Sect. 8.

Given a target $G$, let

$$
\mathcal{G}(t, z):=\left\{v \in \mathcal{A}: Z_{z}^{v}(t) \in G \text { a.s. }\right\},
$$

and

$$
V(t):=\left\{z \in \mathbb{R}^{n} \mid \mathcal{G}(t, z) \neq \varnothing\right\} .
$$

Then, the results of the previous section imply that

Theorem 5.1. $V(t)$ is a viscosity solution of the mean curvature flow as defined in [4], i.e., the characteristic function of the complement of $V(t)$ is a discontinuous viscosity solution of (5.4). 
Example 5.2. We consider the evolution of spheres discussed in Example 5.1. Then, it follows from Remark 4.1 that $V(t)$ is nonempty for all $t$ sufficiently close to $T$. Indeed, we may obtain from (4.5) an explicit lower bound for $V(t)$ in terms of $C$ and $R(T)$.

Example 5.3. Again, in the context of Example 5.1, we now construct an optimal control process. Indeed in that example $G=\partial B_{R_{0}}$. Suppose that $z \in V(T)$. Then, there exists an adapted process $v(\cdot) \in S^{n-1}$, such that the corresponding state process $Z_{z}^{v}$ satisfies, $Z_{z}^{v}(T) \in G$, or equivalently, $\left|Z_{z}^{v}(T)\right|=R_{0}$. We apply Itô's rule to $\left|Z_{z}^{v}(t)\right|^{2}$,

$\left(R_{0}\right)^{2}=\left|Z_{z}^{v}(T)\right|^{2}=|z|^{2}+2(n-1) T+2 \sqrt{2} \int_{0}^{T}\left(Z_{z}^{v}(t)\right)^{*}\left(I_{n}-v(t) v^{*}(t)\right) d W(t)$.

Since the above identity holds almost surely, we conclude that the stochastic integral has to be equal to zero. Hence

$$
v(t)= \pm \frac{Z_{z}^{v}(t)}{\left|Z_{z}^{v}(t)\right|},
$$

and $|z|=R(T):=(R(0))^{2}-2(n-1) T$. Therefore, $V(T) \subset \partial B_{R(T)}$. Also starting from any point $z \in \partial B_{R(T)}$ if we use the feedback control $v$ as above, then the above calculation implies that the corresponding state process $Z_{z}^{v}$ satisfies $\left|Z_{z}^{v}(T)\right|=R(0)$. Hence, $V(T)=\partial B_{R(T)}$.

In this example, we have shown the equivalence between the stochastic target problem and the mean curvature flow by explicitely constructing the control process. Such a construction is also possible when the mean curvature flow has a smooth solution. In this case the control process is constructed by using the gradient of the distance function; [28].

\subsection{Codimension $k$ mean curvature flow}

A slight change of the above problem gives the codimension $k$ mean curvature flow. For an integer $k<n$ consider the state process

$$
d Z_{z}^{v}(s)=\sqrt{2}\left[I_{n}-\sum_{j=1}^{k} v_{j}(s) v_{j}^{*}(s)\right] d W(s),
$$

where the control set is defined by:

$$
U=\left\{v=\left(v_{1}, \ldots, v_{k}\right) \in\left(S^{n-1}\right)^{k}: v_{i}^{*} v_{j}=0 \text { for all } 1 \leq i \neq j \leq k\right\} .
$$

The control set $U$ can be seen as the Grassmanian manifold of $n-k$ dimensional unoriented planes. Indeed, for $v \in U$, let $\Pi^{v}$ be the projection matrix onto a plane orthogonal to $v$, i.e.

$$
\Pi^{v}:=I_{n}-\sum_{i=1}^{k} v_{i} v_{i}^{*}
$$


For $p \neq 0$,

$$
\mathcal{N}(t, z, p)=\mathcal{N}(p)=\left\{v \in U \mid \Pi^{v} p=0\right\} .
$$

Therefore, (4.1) is satisfied and the dynamic programming equation is

$$
u_{t}(t, z)+F\left(D^{2}(t, z), D u(t, z)\right)=0,
$$

where

$$
F(A, p)=\sup _{\nu \in \mathcal{N}(p)}\left\{- \text { trace }\left[A \Pi^{\nu}\right]\right\} .
$$

We claim that the nonlinear function $F$ is equal to $F_{k}$ defined in (5.5). Thus an analogue of Theorem 5.1 holds for codimension $k$ mean curvature flow.

Proposition 5.1. For all $p \in \mathbb{R}^{n}, p \neq 0$, and $n \times n$ symmetric matrix $A$,

$$
F(A, p)=F_{k}(A, p)=-\sum_{j=1}^{n-k} \lambda_{j}^{p}(A) .
$$

Proof. For an $n \times n$ symmetric matrix $M$, let $\lambda_{1}(M) \leq \ldots \leq \lambda_{n}(M)$ be the ordered eigenvalues of $M$. Also, for $v=\left(v_{1}, \ldots, v_{k}\right) \in U, \lambda_{1}^{v}(M) \leq \ldots \leq$ $\lambda_{n-k}^{v}(M)$ denote the ordered eigenvalues of $\Pi^{v} M \Pi^{v}$ corresponding to eigenvectors orthogonal to $v_{1}, \ldots, v_{k}$.

Since $A \Pi^{v}=A \Pi^{v} \Pi^{v}, \Pi^{v}$ is symmetric, and since all $v_{j}$ is an eigenvector of $\Pi^{v} A \Pi^{v}$ with zero eigenvalue,

$$
\operatorname{trace}\left[A \Pi^{\nu}\right]=\operatorname{trace}\left[\Pi^{v} A \Pi^{\nu}\right]=\sum_{j=1}^{n} \lambda_{j}\left(\Pi^{v} A \Pi^{\nu}\right)=\sum_{j=1}^{n-k} \lambda_{j}^{\nu}(A) .
$$

We now claim that for $v \in \mathcal{N}(p), \lambda_{i}^{\nu}(A) \geq \lambda_{i}^{p}(A)$ for every $i=1, \ldots, n-k$. Indeed, $\lambda_{i}^{\nu}$ admits a representation

$$
\lambda_{i}^{v}(A)=\sup _{\eta \in K_{i}} \inf \left\{\Pi^{v} A \Pi^{v} x \cdot x|| x \mid=1, \Pi^{v} x=x, \Pi^{\eta} x=x\right\},
$$

where $K_{1}=\emptyset$, and for $i>1, K_{i}$ is set of all (i-1) mutually orthogonal, unit vectors $\eta=\left\{\eta_{1}, \ldots, \eta_{i-1}\right\}$.

For $v \in \mathcal{N}(p)$, and $x$ satisfying $\Pi^{v} x=x$,

$$
\Pi^{v} A \Pi^{v} x=\Pi^{p} A \Pi^{p} x=A x .
$$

Hence

$$
\begin{aligned}
\lambda_{i}^{v}(A) & =\sup _{\eta \in K_{i}} \inf \left\{\Pi^{v} A \Pi^{v} x \cdot x|| x \mid=1, \Pi^{v} x=x, \Pi^{\eta} x=x\right\} \\
& =\sup _{\eta \in K_{i}} \inf \left\{\Pi^{p} A \Pi^{p} x \cdot x|| x \mid=1, \Pi^{v} x=x, \Pi^{\eta} x=x\right\} \\
& \geq \sup _{\eta \in K_{i}} \inf \left\{\Pi^{p} A \Pi^{p} x \cdot x|| x \mid=1, \Pi^{p} x=x, \Pi^{\eta} x=x\right\} \\
& =\lambda_{i}^{p}(A) .
\end{aligned}
$$


Conversely, let $e_{i}$ be an eigenvector corresponding to the eigenvalue $\lambda_{i}^{p}$, i.e.,

$$
e_{i} \cdot p=0, \quad \text { and } \quad \Pi^{p} A \Pi^{p} e_{i}=\lambda_{i}^{p}(A) e_{i} .
$$

Set $F:=\left\{e_{1}, \ldots, e_{n-k}\right\}^{\perp}$. By definition, $p \in F$. Let $\hat{v}=\left(\hat{v}_{1}, \ldots, \hat{v}_{k}\right)$ be an orthonormal basis of $F$. Then, $\hat{v} \in \mathcal{N}(p)$, and

$$
\Pi^{\hat{v}} A \Pi^{\hat{v}} e_{i}=\Pi^{p} A \Pi^{p} e_{i}=\lambda_{i}^{p}(A) e_{i} .
$$

Hence

$$
\begin{aligned}
F(A, p) & \geq-\operatorname{trace}\left[A \Pi^{\hat{v}}\right]=-\sum_{j=1}^{n-k} \lambda_{j}^{\hat{v}}(A) \geq-\sum_{j=1}^{n-k} \lambda_{j}^{p}(A) \\
& =F_{k}(A, p) .
\end{aligned}
$$

\section{Application to financial mathematics}

In this section, we discuss three examples from financial mathematics. In all these examples, the dynamic programming principle is used to characterize the reachability set by a Hamilton-Jacobi-Bellman equation. As in Theorem 4.1, this equation holds in the viscosity sense.

\subsection{Stochastic target problem in a jump-diffusion model}

Let $W=\left(W^{1}, \ldots, W^{I}\right)$ be an $I$-dimensional Brownian motion on the probability space $(\Omega, \mathcal{F}, P)$, and $v^{j}(d t, d z)_{j=1, \ldots, J}$ be integer valued random measures defined on the same probability space. We assume that these random measures are constructed by the marked point process $\left(N^{j},\left\{Y_{n}^{j}, n \in \mathbb{I}\right\}\right)$, i.e. $\left\{N^{j}(t) t \geq 0\right\}$ is a counting process corresponding to the exponential random times $\left\{T_{n}^{j}, n \in \mathbb{N}\right\}$, and $\left\{Y^{j}(n), n \in \mathbb{N}\right\}$ is a sequence of random variables with values in the mark space $E$, a Borel subset of $\mathbb{R}_{+}$. Then, $v^{j}$ is given by

$$
v^{j}([0, t] \times B)=\sum_{n \geq 1} \mathbf{1}_{\left\{T_{n}^{j} \leq t\right\}} \mathbf{1}_{B}\left(Y_{n}^{j}\right) \quad \text { for all } t \in[0, T] \text { and } B \in \mathcal{B}_{E} .
$$

As discussed in Sect. 2.5, we assume that $\mathcal{F}$ is the $P$ completion of the $\sigma$ algebra generated by the Brownian motion and the random measures. We also let $\mathbb{F}=\{\mathcal{F}(t), 0 \leq t \leq T\}$ be the $P$-completed filtration generated by the random measures $v^{j}(d t, d z)$ and the Brownian motion $W$.

The random measure $v(d t, d z)=\left(v^{1}, \ldots, v^{J}\right)$ is assumed to have a predictable intensity kernel $m_{t}(d z)$ with

$$
E\left[\sup _{0 \leq t \leq T} \int_{E} m_{t}(d z)\right]<\infty .
$$


In particular, this means that there is a finite number of jumps during any finite time interval. By definition of the intensity kernel $m_{t}(d z)$, the compensated jump process:

$$
\tilde{v}(d t, d z)=v(d t, d z)-m_{t}(d z) d t
$$

is such that $\{\tilde{v}([0, t] \times B), 0 \leq t \leq T\}$ is a $(P, \mathbb{F})$ martingale for all $B \in \mathcal{B}_{E}$.

The control set $\mathcal{A}$ is the collection of all $L^{2}((0, T) \times \Omega)$ adapted processes valued in some closed subset $U$ of $\mathbb{R}^{d}$. Observe that Property A1 is trivially satisfied. Also, in view of Sect. 2.5, the control set $\mathcal{A}$ is a separable metric space, and therefore Property A2 holds by Lemma 2.1.

Given an initial data $(t, z) \in S=[0, T] \times \mathbb{R}^{n}$, and a control $v \in \mathcal{A}$, the controlled process $Z_{t, z}^{v}$ is the unique solution of

$$
\begin{aligned}
d Z_{t, z}^{v}(t)=\mu\left(t, Z_{t, z}^{v}(t), v(t)\right) d t & +\sigma\left(t, Z_{t, z}^{v}(t), v(t)\right) d W(t) \\
& +\int_{E} \gamma\left(t, Z_{t, z}^{v}(t), v(t), e\right) v(d t, d e),
\end{aligned}
$$

and the condition $Z_{t, z}^{v}(t)=z$. We set $Z_{t, s}^{v}(r)=0$ for $r<t$. It is well known that the above stochastic differential equation has a unique strong solution under some conditions on the coefficients $\mu, \sigma$ and $\gamma$.

The target set in this example is given by

$$
G:=\mathcal{E} \operatorname{pi}(g)=\left\{z=(x, y) \in \mathbb{R}^{n-1} \times \mathbb{R}: y \geq g(x)\right\}
$$

for some measurable function $g: \mathbb{R}^{n-1} \longrightarrow \mathbb{R}$. Then, the stochastic target problem is

$$
V(t):=\left\{z \in \mathbb{R}^{n}: Z_{t, z}^{v}(T) \in G \text { for some } v \in \mathcal{U}\right\}
$$

A straightforward application of Theorem 3.1 yields the following.

Proposition 6.1. Let $\mu(t, z, u), \sigma(t, z, u)$ and $\gamma(t, z, u, e)$ be continuous functions, globally Lipschitz in $(z, u) \in \mathbb{R}^{n} \times U$ uniformly in $(t, e) \in[0, T] \times E$. Then, $V$ satisfies the dynamic programming principle of Theorem 3.1.

Proof. First, observe that the above conditions on the coefficients ensure the existence of a unique strong solution $Z_{\theta, \xi}^{\nu}$ of the stochastic differential equation (6.2) for any given initial condition $\xi \in L_{n}^{2}(\theta)$ and $\theta \in \mathcal{T}$; see for instance [16]. Properties $\mathbf{Z 1}, \mathbf{Z 2}, \mathbf{Z 3}$ and $\mathbf{Z 4}$ are clearly satisfied by the solution $Z_{\theta, \xi}^{v}$. Also Properties A1 and A2 of the control set hold.

We continue with a proof of Property ZZ5. From classical estimates we know that for each $v \in \mathcal{A}$, the map $(t, z) \in S \longmapsto Z_{t, z}^{v}(T) \in L_{n}^{2}$ is continuous (see for instance [16].) So it remains to prove that for fixed initial data $(t, z) \in S$, the map $v \in \mathcal{U} \longmapsto Z_{t, z}^{v}(T) \in L_{n}^{2}$ is continuous uniformly in $(t, z)$.

In the rest of this proof, for the ease of notation we suppress the dependence of the coefficients on the $t$ variable. 
For $v_{1}, v_{2} \in \mathcal{A}$, we directly estimate that

$$
\begin{aligned}
\left|Z_{t, z}^{\nu_{1}}-Z_{t, z}^{\nu_{2}}\right| \leq & \int_{t}^{T}\left|\mu\left(Z_{t, z}^{\nu_{1}}(r), v_{1}(r)\right)-\mu\left(Z_{t, z}^{\nu_{2}}(r), \nu_{2}(r)\right)\right| d r \\
& +\left|\int_{t}^{T}\left(\sigma\left(Z_{t, z}^{\nu_{1}}(r), \nu_{1}(r)\right)-\sigma\left(Z_{t, z}^{\nu_{2}}(r), \nu_{2}(r)\right)\right) d W(r)\right| \\
& +\left|\int_{t}^{T} \int_{E}\left(\gamma\left(Z_{t, z}^{\nu_{1}}(r), \nu_{1}(r), e\right)-\gamma\left(Z_{t, z}^{\nu_{2}}(r), \nu_{2}(r), e\right)\right) v(d r, d e)\right| .
\end{aligned}
$$

The global Lipschitz property of the coefficients in $(z, u)$ uniformly in $(t, e)$ together with (6.1), implies that

$$
\begin{aligned}
& E\left|Z_{t, z}^{\nu_{1}}(T)-Z_{t, z}^{\nu_{2}}(T)\right|^{2} \leq \\
& \quad C \int_{t}^{T} E\left|\mu\left(Z_{t, z}^{\nu_{1}}(r), \nu_{1}(r)\right)-\mu\left(Z_{t, z}^{\nu_{2}}(r), \nu_{2}(r)\right)\right|^{2} d r \\
& \quad+C \int_{t}^{T} E\left|\sigma\left(Z_{t, z}^{\nu_{1}}(r), \nu_{1}(r)\right)-\sigma\left(Z_{t, z}^{\nu_{2}}(r), \nu_{2}(r)\right)\right|^{2} d r \\
& \quad+C \int_{t}^{T} E \int_{E}\left|\gamma\left(Z_{t, z}^{\nu_{1}}(r), \nu_{1}(r), e\right)-\gamma\left(Z_{t, z}^{\nu_{2}}(r), \nu_{2}(r), e\right)\right| m_{r}(d e) d r \\
& \leq C\left(\left\|v_{1}-v_{2}\right\|_{I_{n}^{2}}^{2}+\int_{t}^{T} E\left|Z_{t, z}^{\nu_{1}}(r)-Z_{t, z}^{\nu_{2}}(r)\right|^{2} d r\right),
\end{aligned}
$$

where $C$ is a generic constant whose value may vary. By Gronwall's inequality,

$$
E\left|Z_{t, z}^{\nu_{1}}(T)-Z_{t, z}^{\nu_{2}}(T)\right|^{2} \leq C e^{C(T-t)}\left\|v_{1}-v_{2}\right\|_{\mathbb{H}_{n}^{2}}^{2},
$$

proving that the map $v \in \mathcal{U} \longmapsto Z_{t, z}^{v}(T) \in L_{n}^{2}$ is Lipschitz uniformly in $(t, z) \in S$.

Next, let us split the process $Z_{t, z}^{v}$ into two parts $Z_{t, z}^{v}=\left(X_{t, z}^{v}, Y_{t, z}^{v}\right)$, where $Y_{t, z}^{v}$ is the last component of $Z_{t, z}^{v}$ valued in $\mathbb{R}$. Suppose further that the process $X_{t, z}^{v}=X_{t, x}^{v}$ is independent of the initial data $y$. Then, by a comparison theorem for SDE's, the random variable $Y_{t, z}^{v}$ is increasing in $y$. Consequently, the reachability set $V(t)$ is essentially the epigraph of the following value function,

$$
\begin{aligned}
v(t, x) & :=\inf \{y \in \mathbb{R}:(x, y) \in V(t)\} \\
& =\inf \left\{y \in \mathbb{R}: Z_{t, x, y}^{v}(T) \in \mathcal{E} \operatorname{pi}(g) \text { for some } v \in \mathcal{A}\right\} .
\end{aligned}
$$

The above stochastic target problem was studied by Soner and Touzi [27] in the diffusion case, and extended to this context by Bouchard [5].

This problem is closely related to the theory of forward-backward stochastic differential equations and it is motivated by applications from finance; see e.g. Karatzas and Shreve [18] and Cvitanić and Ma [10]. Previously, convex duality was used to reduce it to a standard stochastic control problem; see Föllmer and Kramkov [14] for the general semimartingale case, and the references therein. Once 
the reduction is proved, then the Hamilton-Jacobi-Bellman equation associated to the problem is derived by means of a classical dynamic programming on the dual problem; see Broadie, Cvitanić and Soner [7] and Cvitanić, Pham and Touzi [11]. Corollary 6.1 below allows to derive the HJB equation directly from the initial problem, and therefore avoids the passage by duality.

In [27], the following dynamic programming principle has been introduced (with only a partial proof), and used successfully in order to obtain a characterization of the value function $v$ by means of the associated Hamilton-Jacobi-Bellman equation in the viscosity sense.

Corollary 6.1. Let the conditions of Proposition 6.1 hold. Suppose further that the process $X_{t, x, y}^{v}=X_{t, x}^{v}$ is independent of $y$. Then, for all stopping time $\theta \geq t$ in $\mathcal{T}$,

$$
v(t, x)=\inf \left\{y \in \mathbb{R}: Z_{t, x, y}^{v}(\theta) \in \mathcal{E} p i(v(\theta, \cdot)) \text { for some } v \in \mathcal{A}\right\} .
$$

Proof. Let $w(t, x)$ denote the right-hand side of the above dynamic programming equation. Clearly, the process $Y_{t, x, y}^{v}$ is strictly increasing in the initial condition $y$ since $X_{t, x, y}^{v}=X_{t, x}^{v}$ is independent of $y$. Then, for all $y>w(t, x)$ and $\varepsilon>0$, we have $Z_{t, x, y+\varepsilon}^{v}(\theta) \in V(\theta)$. From Proposition 6.1, this proves that $(x, y+\varepsilon) \in V(t)$, and therefore $y+\varepsilon \geq v(t, x)$. Since $y>w(t, x)$ and $\varepsilon>0$ are arbitrary, this proves that $w(t, x) \geq v(t, x)$.

Conversely, for all $y>v(t, x), Z_{t, x, y}^{v}(T)=Z_{\theta, Z_{t, x, y}^{v}(\theta)}^{v}(T) \in G$ for some $v \in \mathcal{A}$ and therefore $Y_{t, x, y}^{v}(T) \geq v\left(\theta, X_{t, x, y}^{v}(\theta)\right)$. Hence $y \geq w(t, x)$ and the required inequality follows by letting $y$ converge to $v(t, x)$.

Remark 6.1. In [27], the value function $v$ was shown to be a (discontinuous) supersolution (resp. subsolution) of the associated Hamilton-Jacobi-Bellman equation by means of DP1 (resp. DP2), where:

DP1 Let $(t, x, y) \in S$, and $v \in \mathcal{A}$ be such that $Z_{t, x, y}^{v}(T) \in \mathcal{E} \operatorname{pi}(g)$. Then $Z_{t, x, y}^{v}(\theta) \in$ $\mathcal{E} \operatorname{pi}(v(\theta, \cdot))$ for all stopping time $\theta \in \mathcal{T}$.

DP2 For $(t, x) \in[0, T) \times \mathbb{R}^{n-1}$, set $y^{*}:=v(t, x)$. Then, for all $\eta>0, v \in \mathcal{A}$, and stopping time $\theta \in \mathcal{T}$, we have $P\left[Z_{t, x, y^{*}-\eta}^{v}(\theta) \in \mathcal{E}\right.$ pi $\left.(v(\theta, \cdot))\right]<1$.

Clearly, DP1 and DP2 follow easily from the dynamic programming principle stated in Corollary 6.1.

We conclude the discussion of this example by relating the value function of the stochastic target problem $v(t, x)$ to

$$
\tilde{v}_{\theta, \xi}:=\operatorname{essinf}\left\{\zeta \in L^{2}(\theta): Z_{\theta, \xi, \zeta}^{v}(T) \in \mathcal{E} \operatorname{pi}(g) \text { for some } v \in \mathcal{A}\right\},
$$

for a stopping time $\theta \in \mathcal{T}$ and $\xi \in L^{2}(\theta)$. By the definition of the essential infimum, we obviously have $v(\theta(\omega), \xi(\omega)) \leq \tilde{v}_{\theta, \xi}(\omega)$ for a.e. $\omega \in \Omega$. The measurable selection argument of Lemma 3.1 allows us to prove that equality holds.

Proposition 6.2. Let $\theta \in \mathcal{T}$ and $\xi \in L^{2}(\theta)$ be such that $\tilde{v}_{\theta, \xi} \in L^{2}(\theta)$. Assume further that $v$ is bounded from below. Then,

$$
v(\theta, \xi)=\tilde{v}_{\theta, \xi} \quad P-\text { a.s. }
$$


Proof. We only need to prove that $v(\theta, \xi) \geq \tilde{v}_{\theta, \xi}$ for all $\theta \in \mathcal{T}$ and $\xi \in L_{n-1}^{2}(\theta)$. Fix $\theta \in \mathcal{T}$. Consider the function $f$ mapping $L_{n-1}^{2}(\theta) \times\left(L_{1}^{2}(\theta) \times \mathcal{U}\right)$ into $L_{1}^{2}(\theta)$,

$$
f(\xi, \zeta, v)=\zeta \text { for all }(\xi, \zeta, v) \in L_{n-1}^{2}(\theta) \times\left(L_{1}^{2}(\theta) \times \mathcal{U}\right) .
$$

Set $D_{\xi}:=\left\{(\zeta, \nu) \in L_{1}^{2}(\theta) \times \mathcal{U}: Z_{\theta, \xi, \zeta}^{v}(T) \in G\right\}$. Notice that $D_{\xi} \neq \emptyset$ since $\tilde{v}_{\theta, \xi} \in L^{2}(\theta)$. Then,

$$
v(\theta, \xi)=\inf _{(\zeta, v) \in D_{\xi}} f(\xi, \zeta, \nu) .
$$

Fix $\varepsilon>0$. Since $v(\theta, \xi)$ is bounded from below, it follows from Bertsekas and Shreve [6], Proposition 7.50, that there exists an analytically measurable function $\varphi: L^{2}(\theta) \times\left(L^{2}(\theta) \times \mathcal{A}\right) \longrightarrow L^{2}(\theta)$ such that $(\xi, \varphi(\xi)) \in L^{2}(\theta) \times\left(L^{2}(\theta) \times \mathcal{A}\right)$, and

$$
\zeta:=f(\xi, \varphi(\xi)) \leq v(\theta, \xi)+\varepsilon .
$$

Using the notion of universal $\sigma$-algebra as in the proof of Lemma 3.1, we see that, for all probability measure $\mu$ on $\mathbb{R}^{n-1}$, there exists a Borel measurable function $\zeta_{\mu}$ such that $\zeta=\zeta_{\mu} P$-a.s.

For a positive integer $n$, set $\zeta_{n}:=\zeta_{\mu}+n^{-1}$. In view of the definition of the value function $v,\left(\xi, \zeta_{n}\right) \in V(\theta)$. Using the same measurable selection argument as in the proof of Theorem 3.1, we construct an admissible control $v_{n} \in \mathcal{A}$ such that $Z_{\theta, \xi, \zeta_{n}}^{v_{n}}(T) \in \mathcal{E} \operatorname{pi}(g)$. Then, by the definition of $\tilde{v}, \zeta_{n} \geq \tilde{v}_{\theta, \xi}$. We now complete the proof by sending $n$ to infinity and $\varepsilon$ to zero.

\subsection{Super-replication under Gamma constraints}

Let $W=\{W(t), 0 \leq t \leq T\}$ be a Brownian motion on $\mathbb{R}$. The controls are taken from the set $\mathcal{U}$ defined as the collection of adapted processes $v=(\alpha, \gamma)$ in $\mathbb{H}_{2}^{2}$. Given such a control, the state process $Z=(S, X, Y)$ is defined by the initial data $Z_{t, s, x, y}^{v}(t)=\left(S_{t, s}, X_{t, s, x, y}^{v}, Y_{t, y}^{v}\right)(t)=(s, x, y)$ and:

$$
\begin{aligned}
d S_{t, s}(r) & =S_{t, s}(r) \sigma\left(t, S_{t, s}(r)\right) d W(r) \\
d X_{t, s, x, y}^{v}(r) & =Y_{t, y}^{v}(r) d S_{t, s}(r) \\
d Y_{t, y}^{v}(r) & =\alpha(r) d r+\gamma(r) d W(r) .
\end{aligned}
$$

For two constants $-\infty \leq \underline{\Gamma} \leq \bar{\Gamma} \leq+\infty$, the control set is

$$
\mathcal{A}:=\{v=(\alpha, \gamma) \in \mathcal{U}: \underline{\Gamma} \leq \gamma(t) \leq \bar{\Gamma}\} .
$$

In this example, the target is given by

$$
G:=\left\{z=(s, x, y) \in \mathbb{R}^{3} \times \mathbb{R}: x \geq g(s)\right\}
$$

for some measurable function $g: \mathbb{R} \longrightarrow \mathbb{R}$, and the target reachability problem is defined by:

$$
V(t):=\left\{z \in \mathbb{R}^{3}: Z_{t, z}^{v}(T) \in G \text { for some } v \in \mathcal{A}\right\} .
$$


In financial mathematics, this control problem arises in the following optimal investment problem in a financial market consisting of one risky asset and a nonrisky asset with price process normalized to unity. $S$ is the price process of a risky asset. The process $Y$ describes the number of shares of risky assets held by an investor at each time $t$. The process $X$ represents the value of the investor's portfolio under the self-financing condition. In the above model, the unbounded variation part of the portfolio process is constrained in the interval $[\underline{\Gamma}, \bar{\Gamma}]$. This problem has been first raised by Broadie, Cvitanić and Soner [7], and studied by Soner and Touzi [26] in the case $\underline{\Gamma}=-\infty$. The case $\underline{\Gamma}>-\infty$ is an open problem.

Proceeding as in Proposition 6.1, we obtain the following corollary of Theorem 3.1 .

Proposition 6.3. Let the function $s \sigma(t, s)$ be continuous and globally Lipschitz in $s \in[0, \infty)$ uniformly in $t \in[0, T]$. Then $V$ satisfies the dynamic programming principle of Theorem 3.1.

Next, as in the previous example, we can reduce the problem to a scalar function,

$$
\begin{aligned}
v(t, s, y) & :=\inf \{x \in \mathbb{R}:(s, x, y) \in V(t)\} \\
& =\inf \left\{x \in \mathbb{R}: X_{t, s, x, y}^{v}(T) \geq g\left(S_{t, s}(T)\right) \text { for some } v \in \mathcal{A}\right\} .
\end{aligned}
$$

We then have the following consequence of Proposition 6.3. The proof is omitted since it follows from the same argument as in the proof of Corollary 6.1.

Corollary 6.2. Let the conditions of Proposition 6.3 hold. Then, for any stopping time $\theta \geq \operatorname{tin} \mathcal{T}$, we have:

$$
\begin{aligned}
v(t, s, y) & =\inf \left\{x \in \mathbb{R}: Z_{t, s, x, y}^{v}(\theta) \in \mathcal{E} p i(v(\theta, \cdot))\right\} \\
& =\inf \left\{x \in \mathbb{R}: X_{t, s, x, y}^{v}(\theta) \geq v\left(\theta, S_{t, s}(\theta), Y_{t, y}(\theta)\right)\right\}
\end{aligned}
$$

However, the control problem $v(t, s, y)$ is not the relevant problem in practice, since the number of shares $y$ held at the time origin $t$ is an additional control for the investor. The problem of super-replication under Gamma constraint, as introduced in [26], is defined by:

$$
u(t, s):=\inf _{y \in \mathbb{R}} v(t, s, y)
$$

Unfortunately, the dynamic programming equation does not translate to the value function $u$. However, suppose that the drift term $\alpha(t) d t$ is extended to $d A(t)$ where $A$ is a (new) control in the class of bounded variation processes. Then, clearly, the value function $v(t, s, y)$ does not depend on $y$, since the process $Y$ can jump at time zero by the action of the bounded variation process $A$, and $u(t, s)=v(t, s, y)$ for all $y \in \mathbb{R}$. Under this "relaxation" the value function $u(t, s)$ satisfies the dynamic programming equation of Corollary 6.2. 


\subsection{Super-replication under transaction costs}

The financial market consists of one bank account, with constant price process $S^{0}$, normalized to unity, and $d$ risky assets $S=\left(S^{1}, \ldots, S^{d}\right)$ defined by the dynamics:

$$
S_{t, s}(t)=s \quad \text { and } \quad d S_{t, s}(t)=\operatorname{diag}\left[S_{t, s}(t)\right]\left(b\left(t, S_{t, s}(t)\right) d t+\sigma\left(t, S_{t, s}(t)\right) d W(t)\right) .
$$

Here $W$ is an $d$-dimensional Brownian motion defined on the filtered probability space $(\Omega, \mathcal{F}, \mathbb{F}, P)$, and $b, \sigma$ are coefficients, with the appropriate size, satisfying the usual global Lipschitz condition.

Proportional transaction costs in this financial market are described by the matrix $\lambda=\left(\lambda^{i j}, 0 \leq i, j \leq d\right)$ with non-negative entries. This means that transfers from asset $i$ to asset $j$ are subject to proportional transaction costs $\lambda^{i j}$ for all $i, j=0, \ldots, d$.

The Control set. A trading strategy is an $d+1$-matrix valued process $L$, with initial value $L(0-)=0$, such that $L^{i j}$ is $I F$-adapted, right-continuous, and nondecreasing for all $i, j=0, \ldots, d$. Here, $L^{i j}$ describes the cumulative amount of funds transferred from asset $i$ to asset $j$. We shall denote by $\mathcal{U}=\mathcal{A}$ the collection of all such trading strategies satisfying

$$
\|L\|^{2}:=\sum_{i, j=0}^{d} E\left[\left(L^{i j}(T)\right)^{2}\right]<\infty .
$$

Controlled process. Given a trading strategy $L$ and a vector $x \in \mathbb{R}^{d+1}$ of initial holdings, the portfolio holdings $X_{t, s, x}^{L}=\left(X_{t, s, x}^{L}{ }^{i}, i=0, \ldots, d\right)$ are defined by the dynamics:

$$
\begin{aligned}
& X_{t, s, x}^{L}(0-)=x \\
& d X_{t, s, x}^{L}(t)^{i}=X_{t, s, x}^{L}(t)^{i} \frac{d S_{t, s}^{i}(t)}{S_{t, s}^{i}(t)}+\sum_{j=0}^{d}\left[d L^{j i}(t)-\left(1+\lambda^{i j}\right) d L^{i j}(t)\right],
\end{aligned}
$$

for all $i=0, \ldots, d$. Hence, the state process in this example is $Z_{t, s, x}^{L}=\left(S_{t, s}, X_{t, s, x}^{L}\right)$ defined by (6.3)-(6.5).

Hedging problem. The solvency region $K$ is given by

$$
K:=\left\{x \in \mathbb{R}^{d+1}: \exists a^{i j} \geq 0, x^{i}+\sum_{j=0}^{d}\left(a^{j i}-\left(1+\lambda^{i j}\right) a^{i j}\right) \geq 0 ; i=0, \ldots, d\right\},
$$

i.e. the collection of portfolio holdings whose liquidation value, through some convienient transfers, is nonnegative. The set $K$ is a closed convex cone containing the origin. It then induces a partial ordering $\succeq$ defined by $x \succeq 0$ iff $x \in K$. 
Given some measurable function $g: \mathbb{R}^{d} \longrightarrow \mathbb{R}^{d+1}$, the target is defined by

$$
G:=\mathcal{E}_{\mathrm{pi}_{\succeq}}(g)=\left\{z=(s, x) \in \mathbb{R}^{d} \times \mathbb{R}^{d+1}: x-g(s) \succeq 0\right\} .
$$

The target reachability problem is defined by:

$$
V(t):=\left\{z \in \mathbb{R}^{d} \times \mathbb{R}^{d+1}: Z_{t, z}^{L}(T) \in G \text { for some } L \in \mathcal{A}\right\} .
$$

An immediate corollary of Theorem 3.1 is the following dynamic programming principle.

Proposition 6.4. Let the coefficients of the model (6.3)-(6.5) be Lipschitz in ( $s, x)$ uniformly in $t$. Then, for all stopping time $\theta \geq t$ in $\mathcal{T}$,

$$
V(t)=\left\{z \in \mathbb{R}^{d} \times \mathbb{R}^{d+1}: Z_{t, z}^{L}(\theta) \in V(\theta) \text { for some } L \in \mathcal{A}\right\} .
$$

Proof. As in the proof of Proposition 6.1, we only need to prove that the map $L \in \mathcal{A} \longmapsto Z_{t, z}^{L}(T) \in L_{2 d+1}^{2}$ is Lipschitz-continuous. We integrate the state equation to obtain

$$
\begin{aligned}
X_{t, s, x}^{L}(r)^{i}=x^{i}+\int_{t}^{r} & X_{t, s, x}^{L}(\rho) \frac{{ }^{i} \frac{d S_{t, s}^{i}(\rho)}{S_{t, s}^{i}(\rho)}}{} \\
& +\sum_{j=0}^{d}\left[\left(L^{j i}(r)-L^{j i}(t)\right)+\left(1+\lambda^{i j}\right)\left(L^{i j}(r)-L^{i j}(t)\right)\right] .
\end{aligned}
$$

For any two control processes $L_{1}, L_{2}$, set

$$
Y(r):=X_{t, s, x}^{L_{1}}(r)^{i}-X_{t, s, x}^{L_{2}}(r)^{i}
$$

so that

$$
\|Y(r)\|_{L_{d+1}^{2}}^{2} \leq C\left[\int_{t}^{r}\|Y(\rho)\|_{L_{d+1}^{2}}^{2} d \rho+\left\|L_{1}-L_{2}\right\|^{2}\right],
$$

where $C$ is an appropriate constant, and $\left\|L_{1}-L_{2}\right\|:=E\left[\operatorname{Var}\left(L_{1}-L_{2}\right)^{2}\right]$, where $\operatorname{Var}\left(L_{1}-L_{2}\right)$ is the variation of the BV process $L_{1}-L_{2}$. Now the continuity follows from Grownwall's inequality.

Finally, we interpret the dynamic programming principle in terms of the hedging set defined by Kabanov [17],

$$
v(t, s):=\left\{x \in \mathbb{R}^{d+1}:(s, x) \in V(t)\right\} .
$$

The following result is an immediate consequence of Proposition 6.4.

Corollary 6.3. Let the conditions of Proposition 6.4 hold. Then, for all stopping time $\theta \geq t$ in $\mathcal{T}$,

$$
v(t, s)=\left\{x \in \mathbb{R}^{d+1}: X_{t, s, x}^{L}(\theta) \in v\left(\theta, S_{t, s}(\theta)\right) \text { for some } L \in \mathcal{A}\right\} .
$$


Proof. Denote by $w(t, x)$ the right-hand side set of the above dynamic programming equation. Let $x$ be in $v(t, s)$. By definition, this means that $(s, x) \in V(t)$. From Proposition 6.4, there exists some $L \in \mathcal{A}$ such that $Z_{t, s, x}^{L}(\theta) \in V(\theta)$, i.e. $X_{t, s, x}^{L}(\theta) \in$ $v\left(\theta, S_{t, s}(\theta)\right)$. Hence $x \in w(t, x)$.

Conversely, if $x \in w(t, s)$, then $Z_{t, s, x}^{L}(\theta) \in V(\theta)$ for some $L \in \mathcal{A}$, and therefore $(s, x) \in V(t)$ by Proposition 6.4. Hence $x \in v(t, s)$.

\section{Extension to stochastic viability}

The target reachability problem discussed previously can be viewed as a stochastic control problem with constrained state process at the terminal time. Our dynamic programming principle can be stated in a slightly more general framework where the state process is constrained at any time $t \in[0, T]$.

Let the state process $Z_{\theta, \xi}^{v}$ be as defined in Sects. 2.2 and 2.3. In Sect. 2.4, we introduced a target $G$ for the terminal value of the state process. Instead, we define here a tube $\left\{G_{t}, 0 \leq t \leq T\right\}$ where $G_{t}$ is a Borel subset of $\mathbb{R}^{d}$ for all $t \in[0, T]$.

Following Aubin et al. [2,3], we shall say that the process $Z$ is viable if $Z(t) \in G_{t}$ for all $t \in[0, T]$. This leads naturally to defining the viability set:

$$
\bar{V}(t):=\left\{z \in \mathbb{R}^{n}: Z_{t, z}^{v} \text { is viable for some } v \in \mathcal{A}\right\} .
$$

This problem has been studied extensively in the deterministic framework, and we refer to Aubin et al. [2,3] for a discussion of the diffusion case.

The following dynamic programming principle follows from the same arguments as in Sect. 3. Therefore, we only provide a formal statement for it.

Theorem 7.1. Let $(t, z) \in S$, and $\theta \geq t$ be a stopping time in $\mathcal{T}$. Then,

$$
\bar{V}(t)=\left\{z \in \mathbb{R}^{n}: Z_{t, z}^{v}(. \wedge \theta) \in G_{. \wedge \theta} \text { and } Z_{t, z}^{v}(\theta) \in V(\theta) \text { for some } v \in \mathcal{A}\right\} \text {. }
$$

We only need the measurability of each $G_{t}$ and the measurability of the tube $\left\{(t, z): z \in G_{t}\right\}$ in the product topology.

In the financial application, this extension is necessary in order to deal with the problem of super-replication of American contingent claims.

\section{Viscosity property}

In this section, we prove Theorem 4.1. We shall first prove that $u$ is a discontinuous supersolution of (4.1) by a similar method to the one developed in our earlier paper [27]. The proof of the subsolution property requires more attention than in [27] because of the singularity of $\mathcal{N}(t, z, p)$ at $p=0$.

Here we only study a certain type of weak solution, distance solutions, as developed by the first author [25], [4]. Other types of weak solutions easily follow from this result and they will be studied in [29].

As in Sect. 4 and Sect. 5, we assume that $Z_{t, z}^{v}$ is a diffusion. We first start by some easy consequences of the main technical condition reported in Assumption 4.1. 
Remark 8.1. Let $F$ be as in (4.2). In Theorem 4.1 we assume that it is locally Lipschitz on $\{(t, z, p): p \neq 0\}$. This continuity assumption is closely related to the behavior of $\mathcal{N}$, and indeed it would follow from a slightly stronger version of Assumption 4.1.

As in the previous sections, let $u$ be the characteristic function of the reachability set $V(t)$, and let $u_{*}$ be the lower-semicontinuous envelope and, respectively, $u^{*}$ be the upper-semicontinuous envelope of $u$

$$
u_{*}(t, x):=\liminf _{\left(t^{\prime}, x^{\prime}\right) \rightarrow(t, x)} u\left(t^{\prime}, x^{\prime}\right) \text { and } u^{*}(t, x):=\limsup _{\left(t^{\prime}, x^{\prime}\right) \rightarrow(t, x)} u\left(t^{\prime}, x^{\prime}\right) .
$$

According to the definition, $u$ is a viscosity solution if and only if $u_{*}$ is a viscosity supersolution and $u^{*}$ is a viscosity subsolution of (4.1). We refer to [9] and [15] for the definition of viscosity solutions. In this definition we use the lower and upper semicontinuous envelope of the nonlinearity $F$ defined in (4.2). In view of our assumptions, $F^{*}=F_{*}=F$ whenever the gradient variable is nonzero. Moreover, since $\mathcal{N}(t, z, 0)=U, F^{*}=F$ everywhere.

\subsection{Proof of the supersolution property of $u_{*}$}

According to the definition, we need to prove that the lower semicontinuous envelope $u_{*}$ of $u$ is a viscosity supersolution of (4.1). So suppose that there are a point $\left(t_{0}, z_{0}\right) \in S$ and a smooth function $\varphi$ satisfying

$$
0=\left(u_{*}-\varphi\right)\left(t_{0}, z_{0}\right)=\min _{S}\left(u_{*}-\varphi\right) .
$$

We need to show that

$$
-\varphi_{t}\left(t_{0}, z_{0}\right)+F^{*}\left(t_{0}, z_{0}, D \varphi\left(t_{0}, z_{0}\right), D^{2} \varphi\left(t_{0}, z_{0}\right)\right) \geq 0 .
$$

Since $F^{*}=F$ and $U$ is compact, this is equivalent to show that

$$
-\mathcal{L}^{\nu_{0}} \varphi\left(t_{0}, z_{0}\right) \geq 0 \text { for some } v_{0} \in \mathcal{N}\left(t_{0}, z_{0}, D \varphi\left(t_{0}, z_{0}\right)\right) .
$$

1. Suppose that $u(t, z)$ is equal to a constant in a (space-time) neighborhood of $\left(t_{0}, z_{0}\right)$. Then,

$$
\varphi_{t}\left(t_{0}, z_{0}\right)=D \varphi\left(t_{0}, z_{0}\right)=0, \quad D^{2} \varphi\left(t_{0}, z_{0}\right) \geq 0,
$$

and (8.1) follows for any $v_{0}$.

2. In view of the previous step, we may assume that $u_{*}\left(t_{0}, z_{0}\right)=0$. Then, there exists a sequence $\left(t_{n}, z_{n}\right)_{n \geq 1}$ converging to $\left(t_{0}, z_{0}\right)$ such that $u\left(t_{n}, z_{n}\right)=$ $u_{*}\left(t_{n}, z_{n}\right)=0$. Hence, $z_{n} \in V\left(t_{n}\right)$. For any stopping time $\theta_{n}>t_{n}$, by the dynamic programming principle, there is an admissible control $v_{n} \in \mathcal{A}$ such that

$$
Z_{t_{n}, z_{n}}^{v_{n}}\left(\theta_{n}\right) \in V\left(\theta_{n}\right) \text {, i.e. } u\left(\theta_{n}, Z_{t_{n}, z_{n}}^{v_{n}}\left(\theta_{n}\right)\right)=0 .
$$

Further, since $u \geq u_{*} \geq \varphi$,

$$
0=u\left(\theta_{n}, Z_{t_{n}, z_{n}}^{v_{n}}\left(\theta_{n}\right)\right) \geq \varphi\left(\theta_{n}, Z_{t_{n}, z_{n}}^{v_{n}}\left(\theta_{n}\right)\right) \quad P-\text { a.s.. }
$$


Set $\beta_{n}=-\varphi\left(t_{n}, z_{n}\right)$ so that by Itô's lemma,

$$
\begin{aligned}
0 \leq & \beta_{n}-\int_{t_{n}}^{\theta_{n}} \mathcal{L}^{v_{n}(s)} \varphi\left(s, Z_{t_{n}, z_{n}}^{v_{n}}(s)\right) d s \\
& -\int_{t_{n}}^{\theta_{n}}\left[\sigma^{*}\left(s, Z_{t_{n}, z_{n}}^{v_{n}}(s), v_{n}(s)\right) D \varphi\left(s, Z_{t_{n}, z_{n}}^{v_{n}}(s)\right)\right]^{*} d W(s) .
\end{aligned}
$$

3. For a large constant $C$, set

$$
\theta_{n}:=\inf \left\{s>t_{n}:\left|Z_{t_{n}, z_{n}}^{v_{n}}(s)\right| \geq C\right\} .
$$

Since $\mu$ and $\sigma$ are bounded and $\left(t_{n}, z_{n}\right) \longrightarrow\left(t_{0}, z_{0}\right)$, by an application of the Borel-Cantelli Lemma

$$
\liminf _{n \rightarrow \infty} t \wedge \theta_{n}>t_{0} P-\text { a.s. for all } t>t_{0} .
$$

We shall report the proof of this claim in the last step of this proof. For $\xi \in \mathbb{R}$, we introduce the probability measure $P_{n}^{\xi}$ equivalent to $P$ defined by the density process:

$$
M_{n}^{\xi}(t):=\mathcal{E}\left(\xi \int_{t_{n}}^{t \wedge \theta_{n}}\left(\sigma^{*} D \varphi\right)\left(s, Z_{t_{n}, z_{n}}^{v_{n}}(s), v_{n}(s)\right)^{*} d W(s)\right) ; \quad t \geq t_{n},
$$

where $\mathcal{E}(\cdot)$ is the Doléans-Dade exponential operator. Observe that the process $M_{n}^{\xi}$ is a martingale, by the definition of $\theta_{n}$. We shall denote by $E_{n}^{\xi}$ the expectation operator under $P_{n}^{\xi}$. By Girsanov Theorem, the process $W(\cdot)-\int_{t_{n}}^{\cdot} \xi(\sigma D \varphi)\left(s, Z_{t_{n}, z_{n}}^{v_{n}}(s)\right.$, $\left.v_{n}(s)\right) d s$ is a Brownian motion under $P_{n}^{\xi}$.

We take the expected value under $P_{n}^{\xi}$ in (8.2). The result is

$$
\begin{aligned}
0 & \leq \beta_{n}-E_{n}^{\xi}\left[\int_{t_{n}}^{t \wedge \theta_{n}}\left(\mathcal{L}^{v_{n}(s)} \varphi\left(s, Z_{t_{n}, z_{n}}^{v_{n}}(s)\right)\right) d s\right] \\
& -\xi E_{n}^{\xi}\left[\int_{t_{n}}^{t \wedge \theta_{n}}\left|\sigma^{*}\left(s, Z_{t_{n}, z_{n}}^{v_{n}}(s), v_{n}(s)\right) D \varphi\left(s, Z_{t_{n}, z_{n}}^{v_{n}}(s)\right)\right|^{2} d s\right],
\end{aligned}
$$

for all $t>t_{n}$. Then, we take the limit as $n$ tends to infinity. Since $\beta_{n}$ converges to zero, the result is the following inclusion. (We refer to [27], Step 2 of Sect. 4.1, for the technical details.)

$$
\liminf _{h \downarrow 0}-\frac{1}{h} \int_{t_{n}}^{t_{n}+h}\left[\mathcal{L}^{v_{n}(s)} \varphi\left(t_{0}, z_{0}\right)-\xi\left|\sigma^{*}\left(t_{0}, z_{0}, v_{n}(s)\right) D \varphi\left(t_{0}, z_{0}\right)\right|^{2}\right] d s \geq 0 .
$$

Set

$$
\mathcal{V}\left(t_{0}, z_{0}\right):=\left\{-\mathcal{L}^{v} \varphi\left(t_{0}, z_{0}\right)-\xi\left|\sigma^{*}\left(t_{0}, z_{0}, v\right) D \varphi\left(t_{0}, z_{0}\right)\right|^{2}: v \in U\right\},
$$

so that for any $h>0$,

$-\frac{1}{h} \int_{t_{n}}^{t_{n}+h}\left[\mathcal{L}^{v_{n}(s)} \varphi\left(t_{0}, z_{0}\right)-\xi\left|\sigma^{*}\left(t_{0}, z_{0}, v_{n}(s)\right) D \varphi\left(t_{0}, z_{0}\right)\right|^{2}\right] d s \in \overline{\operatorname{co}} \mathcal{V}\left(t_{0}, z_{0}\right)$, 
where $\bar{c} \mathcal{V}\left(t_{0}, z_{0}\right)$ is the closed, convex hull of the set $\mathcal{V}\left(t_{0}, z_{0}\right)$. Therefore, it follows that:

$$
0 \leq \sup _{\phi \in \overline{\operatorname{co}} \mathcal{V}} \phi=\sup _{\phi \in \mathcal{V}} \phi=\sup _{\nu \in U}\left\{-\mathcal{L}^{v} \varphi\left(t_{0}, z_{0}\right)-\xi\left|\sigma^{*}\left(t_{0}, z_{0}, v\right) D \varphi\left(t_{0}, z_{0}\right)\right|^{2}\right\}
$$

for all $\xi \in \mathbb{R}$.

4. For a large positive integer $n$, set $\xi=-n$. Since $U$ is compact, the supremum in (8.4) is attained at some $\hat{v}_{n} \in U$ and

$$
-n\left|\sigma^{*}\left(t_{0}, z_{0}, \hat{v}_{n}\right) D \varphi\left(t_{0}, z_{0}\right)\right|^{2}-\mathcal{L}^{\hat{v}_{n}} \varphi\left(t_{0}, z_{0}\right) \geq 0 .
$$

By passing to a subsequence, we may assume that there exists $v_{0} \in U$ such that $\hat{v}_{n} \rightarrow v_{0}$. Now let $n$ to infinity in the last inequality to conclude that

$$
-\mathcal{L}^{\nu_{0}} \varphi\left(t_{0}, z_{0}\right) \geq 0
$$

and that $\sigma^{*}\left(t_{0}, z_{0}, \nu_{0}\right) D \varphi\left(t_{0}, z_{0}\right)=0$. This proves that

$$
\nu_{0} \in \mathcal{N}\left(t_{0}, z_{0}, D \varphi\left(t_{0}, z_{0}\right)\right)
$$

and therefore (8.1) holds.

5. We now turn to a rigorous proof of (8.3), as requested by an anonymous referee. From the dynamics of the controlled process $Z_{t_{n}, z_{n}}^{v_{n}}$ and the boundedness of $\mu$, we have

$$
\left|Z_{t_{n}, z_{n}}^{v_{n}}\left(t \wedge \theta_{n}\right)-z_{n}\right| \leq|\mu|_{\infty}\left(\theta_{n}-t_{n}\right)+\left|\int_{t_{n}}^{\theta_{n}} \sigma(\ldots) d W(t)\right| .
$$

Now suppose that $\liminf _{n \rightarrow \infty} t \wedge \theta_{n}=t_{0}$, for some $t>t_{0}$. Then $\liminf _{n \rightarrow \infty} \theta_{n}=t_{0}$ and $\lim _{k \rightarrow \infty} \theta_{n_{k}}=t_{0}$ for some subsequence $\left(n_{k}\right)$ depending on $\omega$. By taking the limit along $\left(n_{k}\right)$ in the above inequality and using the fact that $t>t_{0},\left(t_{n}, z_{n}\right) \longrightarrow$ $\left(t_{0}, z_{0}\right),|\sigma|_{\infty}<\infty$, we see that

$$
Z_{t_{n_{k}}, z_{n_{k}}}^{v_{n_{k}}}\left(\theta_{n_{k}}\right) \longrightarrow z_{0} \quad \text { a.s. . }
$$

This provides the required contradiction since $\left|Z_{t_{n_{k}}, z_{n_{k}}}^{v_{n_{k}}}\left(\theta_{n_{k}}\right)\right|=C>\left|z_{0}\right|$ for large $k$.

\subsection{Proof of the subsolution property of $u^{*}$}

Suppose that there are a point $\left(t_{0}, z_{0}\right) \in S$ and a smooth test function $\varphi$ satisfying

$$
0=\left(u^{*}-\varphi\right)\left(t_{0}, z_{0}\right)=\max _{t \geq t_{0}, z \in \mathbb{R}^{n}}\left(u^{*}-\varphi\right)(t, z) .
$$


We may assume that the above maximum is strict and that the Hessian $D^{2} \varphi$ has full rank. We need to prove that

$$
-\varphi_{t}\left(t_{0}, z_{0}\right)+F_{*}\left(t_{0}, z_{0}, D \varphi\left(t_{0}, z_{0}\right), D^{2} \varphi\left(t_{0}, z_{0}\right)\right) \leq 0 .
$$

Recall that, $F_{*}(t, z, p, A)=F(t, z, p, A)$ when $p \neq 0$, so that the above inequality reduces to

(8.7) $\sup \left\{-\mathcal{L}^{v} \varphi\left(t_{0}, z_{0}\right): v \in \mathcal{N}\left(t_{0}, z_{0}, D \varphi\left(t_{0}, z_{0}\right)\right)\right\} \leq 0$ when $D \varphi\left(t_{0}, z_{0}\right) \neq 0$.

We consider three cases separately.

Case 1. If $u^{*}\left(t_{0}, z_{0}\right)=0$, then

$$
\varphi\left(t_{0}, z_{0}\right)=0, \quad D \varphi\left(t_{0}, z_{0}\right)=0, \quad D^{2} \varphi\left(t_{0}, z_{0}\right) \geq 0,
$$

and (8.7) follows.

Case 2. Suppose that $u^{*}\left(t_{0}, z_{0}\right)=1$ and $D \varphi\left(t_{0}, z_{0}\right) \neq 0$. Working towards a contradiction, let us assume that (8.7) does not hold. Then,

$$
-\mathcal{L}^{\nu_{0}} \varphi\left(t_{0}, z_{0}\right)>0 \text { for some } v_{0} \in \mathcal{N}\left(t_{0}, z_{0}, D \varphi\left(t_{0}, z_{0}\right)\right) .
$$

1. Since $D \varphi\left(t_{0}, z_{0}\right) \neq 0$ and $\left(t_{0}, z_{0}\right)$ is a strict maximum, for every sufficiently small $\delta>0$, there exists $\beta=\beta(\delta)>0$ so that on

$$
\mathcal{O}=\mathcal{O}_{\delta}:=B_{\delta}\left(z_{0}\right) \times\left[t_{0}, t_{0}+\delta\right]
$$

$D \varphi \neq 0, \mathcal{L}^{\hat{v}(t, z, D \varphi(t, z))} \varphi(t, z) \leq 0$, and

$$
u^{*}(t, z)-\varphi(t, z) \leq-\beta
$$

on the parabolic boundary $\partial_{p} \mathcal{O}$ of $\mathcal{O}$ :

$$
\partial_{p} \mathcal{O}:=\partial B_{\delta}\left(z_{0}\right) \times\left[t_{0}, t_{0}+\delta\right] \cup B_{\delta}\left(z_{0}\right) \times\left\{t_{0}+\delta\right\} .
$$

Here $\hat{v}(t, z, D \varphi(t, z))$ is as defined in Assumption 4.1. When there is no confusion we will simply write $\hat{v}$ for $\hat{v}(t, z, D \varphi(t, z))$.

2. Let $\left(t_{n}, z_{n}\right)_{n \geq 1}$ be a sequence in $S$ such that

$$
\left(t_{n}, z_{n}\right) \longrightarrow\left(t_{0}, z_{0}\right) \text { and } u\left(t_{n}, z_{n}\right) \longrightarrow u^{*}\left(t_{0}, z_{0}\right)=1 .
$$

Since $\varphi\left(t_{n}, z_{n}\right) \longrightarrow \varphi\left(t_{0}, z_{0}\right)=u^{*}\left(t_{0}, z_{0}\right)=1$, we may assume that:

$$
\left|1-\varphi\left(t_{n}, z_{n}\right)\right|<\beta \text { for all } n \geq 1 \text {. }
$$

Now, consider the feedback control $\tilde{v}(t, z):=\hat{v}(t, z, D \varphi(t, z))$. Since $D \varphi\left(t_{0}, z_{0}\right) \neq 0$ on $\mathcal{O}$, and since $\hat{v}$ is locally Lipschitz whenever the $p$-variable is nonzero, $\tilde{v}$ is locally Lipschitz and there is a solution $Z_{n}$ of

$$
d Z_{n}(s)=\mu\left(s, Z_{n}(s), \tilde{v}\left(s, Z_{n}(s)\right)\right) d s+\sigma\left(s, Z_{n}(s), \tilde{v}\left(s, Z_{n}(s)\right)\right) d W(s)
$$


with initial data $Z_{n}\left(t_{n}\right)=z_{n}$, for small $\left(s-t_{n}\right)$. For ease of notation, we write $v_{n}(s):=\tilde{v}\left(s, Z_{n}(s)\right)$. Set

$$
\theta_{n}:=\inf \left\{s \geq t_{n}:\left(s, Z_{n}(s)\right) \notin \mathcal{O}\right\} .
$$

Then, $\theta_{n}$ is a stopping time, and almost surely $t_{n}<\theta_{n} \leq t_{0}+\delta$ by definition of $\mathcal{O}$. Moreover by the continuity of $Z_{n},\left(\theta_{n}, Z_{n}\left(\theta_{n}\right)\right) \in \partial_{p} \mathcal{O}$. Therefore, by (8.8),

$$
u\left(\theta_{n}, Z_{n}\left(\theta_{n}\right)\right) \leq \varphi\left(\theta_{n}, Z_{n}\left(\theta_{n}\right)\right)-\beta .
$$

3. We apply the Itô's rule to $\varphi$ and use the previous step. The result is

$$
\begin{aligned}
u\left(\theta_{n}, Z_{n}\left(\theta_{n}\right)\right) \leq & \varphi\left(\theta_{n}, Z_{n}\left(\theta_{n}\right)\right)-\beta \\
= & \varphi\left(t_{n}, z_{n}\right)-\beta+\int_{t_{n}}^{\theta_{n}} \mathcal{L}^{v_{n}(s)} \varphi\left(s, Z_{n}(s)\right) d s \\
& +\int_{t_{n}}^{\theta_{n}}\left[\sigma^{*}\left(s, Z_{n}(s), v_{n}(s)\right) D \varphi\left(s, Z_{n}(s)\right)\right]^{*} d W(s) .
\end{aligned}
$$

By the definitions of $v_{n}$ and $\theta_{n}, \sigma^{*}\left(s, Z_{n}(s), v_{n}(s)\right) D \varphi\left(s, Z_{n}(s)\right)=0$, and $\mathcal{L}^{v_{n}(s)} \varphi\left(s, Z_{n}(s)\right) \leq 0$ for $s \in\left[t_{n}, \theta_{n}\right]$. In view of (8.10), this shows that $\{0,1\} \ni$ $u\left(\theta_{n}, Z_{n}\left(\theta_{n}\right)\right)<1$. Therefore

$$
u\left(\theta_{n}, Z_{n}\left(\theta_{n}\right)\right)=0 \quad P-\text { a.s. for all } n \geq 1 .
$$

4. The dynamic programming principle of Theorem 3.1 and (8.12) imply that the initial point $Z_{n}\left(t_{n}\right)=z_{n}$ belongs to $V\left(t_{n}\right)$, i.e. $u\left(t_{n}, z_{n}\right)=0$. Since this is true for all $n \geq 1$, this is in contradiction with (8.9).

Case 3. The only remaining case is $u^{*}\left(t_{0}, z_{0}\right)=1$ and $D \varphi\left(t_{0}, z_{0}\right)=0$. Set

$$
A:=D^{2} \varphi\left(t_{0}, z_{0}\right),
$$

and suppose to the contrary that the subsolution property does not hold:

$$
-\varphi_{t}\left(t_{0}, z_{0}\right)+F_{*}\left(t_{0}, z_{0}, 0, A\right)>0,
$$

and let us work towards a contradiction. By the definition of $F_{*}$ and the continuity Assumption 4.1 on $\mathcal{N}$, there exists a map

$$
\hat{v}:[0, T] \times \mathbb{R}^{n} \times \mathbb{R}^{n} \longrightarrow U
$$

so that, by (8.13),

$$
-\varphi_{t}(t, z)-\mu(t, z, \hat{v}(t, z, p))^{*} p-\frac{1}{2} \operatorname{trace}\left[\sigma \sigma^{*}(t, z, \hat{v}(t, z, p)) A\right]>0
$$

in a neighborhood of $\left(t_{0}, z_{0}, 0\right)$.

Note that the coefficients of the SDE (8.11) are not locally Lipschitz, we can not define the process $Z_{n}$ as in the second case. Hence the arguments of the second case do not apply here. 
For the convenience of the reader, we briefly describe the main idea of this step. We argue by contradiction and we prove that the Hessian matrix $A=D^{2} \varphi\left(t_{0}, z_{0}\right)$ has a negative eigenvalue, whenever the subsolution property does not hold at $\left(t_{0}, z_{0}\right)$. This is done in Step 5 below. This property is exploited in Step 6 to define a convenient perturbation $\varphi_{\varepsilon}$ of the test function $\varphi$ so that $D \varphi_{\varepsilon} \neq 0$ at the local maxima of $\left(u^{*}-\varphi_{\varepsilon}\right)$. Once this is achieved, we use the results proved in Case 2 together with a simple limit argument to conclude.

5. In this step, we prove that (8.13) implies that the Hessian matrix $A$ has a negative eigenvalue. Suppose to the contrary that all eigenvalues of the symmetric matrix $A$ are nonnegative, i.e. there are orthonormal unit vectors $e_{i} \in \mathbb{R}^{d}$ such that:

$$
A e_{i}=\lambda_{i} e_{i} \text { for some } \lambda_{i} \geq 0, i=1, \ldots, d \text {. }
$$

Observe that, in view of (8.13)

$$
-\varphi_{t}\left(t_{0}, z_{0}\right)>0
$$

Choose $m_{i}>\lambda_{i}, i=1, \ldots, d$, and set

$$
\Psi(z):=\sum_{i} m_{i}\left[\left(z_{0}-z\right)^{*} e_{i}\right]^{2}, \quad \bar{B}_{\delta}^{\Psi}:=\left\{z \in \mathbb{R}^{d}: \Psi(z) \leq \delta\right\}, \quad B_{\delta}^{\Psi}:=\operatorname{int}\left(B_{\delta}^{\Psi}\right) .
$$

Then there are constants $0<b<-\varphi_{t}\left(t_{0}, z_{0}\right), c_{0}>0$, and $\delta>0$, such that on $\left(t_{0}, t_{0}+\delta\right] \times \bar{B}_{\delta}^{\Psi}$

$$
\begin{aligned}
\varphi(t, z) & \leq \tilde{\varphi}(t, z):=1-b\left(t-t_{0}\right)+\Psi(z) \\
c_{0} & \leq b-\mu(t, z, \hat{v})^{*} D \Psi(z)-\frac{1}{2} \operatorname{trace}\left[\sigma \sigma^{*}(t, z, \hat{v}) D^{2} \Psi(z)\right]
\end{aligned}
$$

where $\hat{v}=\hat{v}(t, z, D \Psi(z))$.

For future use, we note that since $m_{i}>0, \Psi$ generates a norm on $\mathbb{R}^{d}$, and is therefore equivalent to the Euclidean norm.

Next, fix an arbitrary point $(\tilde{t}, \tilde{z})$ in $\left(t_{0}, t_{0}+\delta\right) \times B_{\delta}^{\Psi}$ close to $\left(t_{0}, z_{0}\right)$, and choose $0<\varepsilon<<\delta$ so that

$$
\Psi(\tilde{z}) \geq 4 \varepsilon
$$

Our ultimate goal for the rest of this step is to prove that

$$
u(\tilde{t}, \tilde{z})=0 \text {. }
$$

Since $(\tilde{t}, \tilde{z})$ and $\varepsilon>0$ are arbitrary, this and Remark 4.1 imply that $u\left(t_{0}, z_{0}\right)=0$. But this contradicts with the hypothesis of this case: $u^{*}\left(t_{0}, z_{0}\right)=1$.

Let $\tilde{v}$ be a smooth function satisfying

$$
\tilde{v}(t, z)=\hat{v}(t, z, D \Psi(z)), \quad \text { on } Q:=\left[t_{0}, t_{0}+\delta\right] \times\left(\bar{B}_{\delta}^{\Psi} \backslash B_{\varepsilon}^{\Psi}\right),
$$


and let $\tilde{Z}:=Z_{\tilde{t}, \tilde{z}}^{\tilde{v}}$ be the solution of the state equation with initial data $\tilde{Z}(\tilde{t})=\tilde{z}$, and feedback control $\tilde{v}$. Set

$$
\theta:=\inf \left\{t>\tilde{t}:(t, \tilde{Z}(t)) \notin \partial_{p} Q\right\},
$$

where

$$
\partial_{p} Q:=\left(\left\{t_{0}+\delta\right\} \times\left[\bar{B}_{\delta}^{\Psi} \backslash B_{\varepsilon}^{\Psi}\right]\right) \cup\left(\left[t_{0}, t_{0}+\delta\right] \times \partial\left[\bar{B}_{\delta}^{\Psi} \backslash B_{\varepsilon}^{\Psi}\right]\right) .
$$

For later use, observe that the definition of the feedback control $\tilde{v}$ together with (8.16) imply that:

$$
\mathcal{L}^{\tilde{v}} \tilde{\varphi}(t, \tilde{Z}(t)) \leq c_{0}<0 \quad \text { and } \quad\left(\sigma(\cdot, \cdot, \tilde{v})^{*} D \tilde{\varphi}\right)(t, \tilde{Z}(t))=0 \text { on }(t, \theta \rrbracket .
$$

Also, on the stochastic interval $(t, \theta \rrbracket$

$$
d \Psi(\tilde{Z}(t))=\mathcal{L}^{\tilde{v}(t)} \Psi(\tilde{Z}(t)) d t,
$$

and moreover

$$
\begin{aligned}
\mathcal{L}^{\tilde{v}(t)} \Psi(\tilde{Z}(t))= & \mu(t, \tilde{Z}(t), \tilde{v}(t))^{*} D \Psi(\tilde{Z}(t)) \\
& +\frac{1}{2} \operatorname{trace}\left[\sigma \sigma^{*}(t, \tilde{Z}(t), \tilde{v}(t)) D^{2} \Psi(\tilde{Z}(t))\right] \\
\geq & \mu(t, \tilde{Z}(t), \tilde{v}(t))^{*} D \Psi(\tilde{Z}(t)) \\
\geq & -2 C \sqrt{\Psi(\tilde{Z}(t))}
\end{aligned}
$$

for some constant $C>0$, where we used the fact that $\mu$ is bounded, together with the equivalence between the norm induced by $\Psi$ and the Euclidean norm. By (8.17),

$$
\sqrt{\Psi(\tilde{Z}(t))} \geq 2 \sqrt{\varepsilon}-C(t-\tilde{t}) \text { for } t \in[\tilde{t}, \theta] .
$$

We now have all the ingredients in order to obtain a contradiction. We consider two cases:

Subcase 1. Suppose that $\tilde{Z}(\theta) \notin \partial B_{\varepsilon}^{\Psi}$. Since the maximum in (8.5) is strict, there is a constant $\beta>0$ such that for $(\tilde{t}, \tilde{z})$ sufficiently close to $\left(t_{0}, z_{0}\right)$,

$$
\left(u^{*}-\varphi\right)(\theta, \tilde{Z}(\theta)) \leq-\beta .
$$

Then by (8.15), Itô's Lemma and (8.19),

$$
\begin{aligned}
u^{*}(\theta, \tilde{Z}(\theta)) & \leq-\beta+\varphi(\theta, \tilde{Z}(\theta)) \\
& \leq-\beta+\tilde{\varphi}(\theta, \tilde{Z}(\theta)) \\
& =-\beta+\tilde{\varphi}(\tilde{t}, \tilde{z})+\int_{\tilde{t}}^{\theta} \mathcal{L}^{\tilde{\mathcal{v}}(t)} \tilde{\varphi}(t, \tilde{Z}(t) d t \\
& \leq-\beta+\tilde{\varphi}(\tilde{t}, \tilde{z}) .
\end{aligned}
$$


Since $(\tilde{t}, \tilde{z})$ is sufficiently close to $\left(t_{0}, z_{0}\right), \varphi\left(t_{0}, z_{0}\right)=u^{*}\left(t_{0}, z_{0}\right)=1$, and $u^{*}$ is valued in $\{0,1\}$, the last inequality proves that $u^{*}(\theta, \tilde{Z}(\theta))=0$. We now proceed as in Step 4: by the dynamic programming principle, $\tilde{z} \in V(\tilde{t})$, i.e. $u(\tilde{t}, \tilde{z})=0$ as required in (8.18).

Subcase 2. Suppose that $\tilde{Z}(\theta) \in \partial B_{\varepsilon}^{\Psi}$. By the definition of the test function $\varphi$ and (8.15),

$$
\begin{aligned}
u^{*}(\theta, \tilde{Z}(\theta)) \leq \varphi(\theta, \tilde{Z}(\theta)) & \leq \tilde{\varphi}(\theta, \tilde{Z}(\theta)) \\
& =1-b(\theta-\tilde{t})+\Psi(\tilde{Z}(\theta)) \\
& =1-b(\theta-\tilde{t})+\varepsilon .
\end{aligned}
$$

In view of (8.20)

$$
\sqrt{\varepsilon}=\sqrt{\Psi(\tilde{Z}(\theta))} \geq 2 \sqrt{\varepsilon}-C(\theta-\tilde{t}) .
$$

Hence

Since $b>0$,

$$
-(\theta-\tilde{t}) \leq-\frac{\sqrt{\varepsilon}}{C}
$$

$$
u^{*}(\theta, \tilde{Z}(\theta)) \leq 1+\varepsilon-\frac{b}{C} \sqrt{\varepsilon}
$$

For a sufficiently small $\varepsilon>0$, this implies that $u^{*}(\theta, \tilde{Z}(\theta))=0$ since $u^{*}$ is valued in $\{0,1\}$. Once again we proceed as in Step 4: by the dynamic programming principle, $\tilde{z} \in V(\tilde{t})$, i.e. $u(\tilde{t}, \tilde{z})=0$ as required in (8.18).

Hence we proved that the Hessian $A=D^{2} \phi\left(t_{0}, z_{0}\right)$ has at least one negative eigenvalue.

6. Let $-\lambda$ be a strictly negative eigenvalue of the Hessian matrix $A=$ $D^{2} \varphi\left(t_{0}, z_{0}\right)$, and let $\hat{e}$ be an associated eigenvector:

$$
A \hat{e}=-\lambda \hat{e}, \quad \lambda>0 .
$$

We may chose $\hat{e}$ so that

$$
\alpha:=D \varphi_{t}\left(t_{0}, z_{0}\right)^{*} \hat{e} \geq 0
$$

As in Step 1 , for every sufficiently small $\delta>0$, there exists $\beta(\delta)>0$ so that on $\mathcal{O}=\mathcal{O}_{\delta}$ (8.8) holds.

We are now in a position to define the convenient perturbation $\varphi_{\varepsilon}$ of the test function $\varphi$ so that the arguments of the second case apply to $\varphi_{\varepsilon}$. For $\varepsilon>0$, set

$$
\varphi_{\varepsilon}(t, z):=\varphi(t, z)+\varepsilon \hat{e}^{*}\left(z-z_{0}\right) .
$$

Then there is $\varepsilon(\delta)>0$ so that (8.8) still holds for all $\varepsilon \leq \varepsilon(\delta)$. Hence, the difference $u^{*}-\varphi_{\varepsilon}$ attains an local maximum in $\mathcal{O}$, say at $\left(t_{\varepsilon}, z_{\varepsilon}\right) \in \mathcal{O}$ :

$$
\left(u^{*}-\varphi\right)\left(t_{\varepsilon}, z_{\varepsilon}\right)=\max _{\mathcal{O}}\left(u^{*}-\varphi\right) .
$$

It is clear that, as $\varepsilon$ tends to zero, the sequence $\left(t_{\varepsilon}, z_{\varepsilon}\right)_{\varepsilon}$ converges to $\left(t_{0}, z_{0}\right)$. 
We shall prove in the next step that

$$
D \varphi_{\varepsilon}\left(t_{\varepsilon}, z_{\varepsilon}\right) \neq 0 \text { for sufficiently small } \varepsilon>0 .
$$

Then we may use the result proved in the second case to conclude that

$$
-\left(\varphi_{\varepsilon}\right)_{t}\left(t_{\varepsilon}, z_{\varepsilon}\right)+F\left(t_{\varepsilon}, z_{\varepsilon}, D \varphi_{\varepsilon}\left(t_{\varepsilon}, z_{\varepsilon}\right), D^{2} \varphi_{\varepsilon}\left(t_{\varepsilon}, z_{\varepsilon}\right)\right) \leq 0 .
$$

We then obtain (8.6) by sending $\varepsilon$ to zero.

7. It remains to prove (8.23). Suppose that $D \varphi_{\varepsilon}\left(t_{\varepsilon_{k}}, z_{\varepsilon_{k}}\right)=0$ for some sequence $\left(\varepsilon_{k}\right)_{k}$ converging to zero, and let us work towards a contradiction. Since $A=$ $D^{2} \varphi\left(t_{0}, z_{0}\right)$ has full rank, for a given $t_{\varepsilon} \geq t_{0}$ the equation

$$
D \varphi_{\varepsilon}\left(t_{\varepsilon}, z_{\varepsilon}\right)=0 \Longleftrightarrow D \varphi\left(t_{\varepsilon}, z_{\varepsilon}\right)=-\varepsilon \hat{e}
$$

has a smooth solution $z_{\varepsilon}$ which is also smooth in $\varepsilon$. We differentiate the above equation with respect to $\varepsilon$ and then evaluate it at $\varepsilon=0$. The result is

$$
\left[\frac{d z_{\varepsilon}}{d \varepsilon}\right]_{\varepsilon=0}=A^{-1}\left(-\hat{e}-D \varphi_{t}\left(t_{0}, z_{0}\right)\left[\frac{d t_{\varepsilon}}{d \varepsilon}\right]_{\varepsilon=0}\right) .
$$

Note that $t_{\epsilon} \geq t_{0}$ for every $\epsilon>0$ by definition of $\mathcal{O}$, and therefore $\left[\frac{d t_{\varepsilon}}{d \varepsilon}\right]_{\varepsilon=0} \geq 0$. Since $A^{-1} \hat{e}=-\lambda^{-1} \hat{e}$, it follows that

$$
\begin{aligned}
\hat{e}^{*}\left[\frac{d z_{\varepsilon}}{d \varepsilon}\right]_{\varepsilon=0} & =\hat{e}^{*} A^{-1}\left[-\hat{e}-D \varphi_{t}\left(t_{0}, z_{0}\right)\left[\frac{d t_{\varepsilon}}{d \varepsilon}\right]_{\varepsilon=0}\right] \\
& =\frac{1}{\lambda}\left[|\hat{e}|^{2}+\alpha\left[\frac{d t_{\varepsilon}}{d \varepsilon}\right]_{\varepsilon=0}\right] \\
& >0
\end{aligned}
$$

Therefore for sufficiently small $\varepsilon>0$, we have $\hat{e}^{*}\left(z_{\varepsilon}-z_{0}\right)>0$, and

$$
\begin{aligned}
\left(u^{*}-\varphi_{\varepsilon}\right)\left(t_{\varepsilon}, z_{\varepsilon}\right) & =\left(u^{*}-\varphi\right)\left(t_{\varepsilon}, z_{\varepsilon}\right)-\varepsilon \hat{e}^{*}\left(z_{\varepsilon}-z_{0}\right) \\
& \leq\left(u^{*}-\varphi\right)\left(t_{0}, z_{0}\right)-\varepsilon \hat{e}^{*}\left(z_{\varepsilon}-z_{0}\right) \\
& =\left(u^{*}-\varphi_{\varepsilon}\right)\left(t_{0}, z_{0}\right)-\varepsilon \hat{e}^{*}\left(z_{\varepsilon}-z_{0}\right) \\
& <\left(u^{*}-\varphi_{\varepsilon}\right)\left(t_{0}, z_{0}\right)
\end{aligned}
$$

which is in contradiction with (8.22).

\section{References}

1. Ambrosio L., Soner H.M. (1996). Level set approach to mean curvature flow in arbitrary codimension. J. Differential Geom. 43, 693-737

2. Aubin J.P., Da Prato G. (1998). The viability theorem for stochastic differential inclusions. Stochastic Anal. Appl. 16, 1-15

3. Aubin J.P., Da Prato G., Frankowska H. (2000). Stochastic invariance for differential inclusions. Set-Valued Analysis (to appear) 
4. Barles G., Soner H.M., Souganidis P.E. (1993). Front propagation and phase field theory. SIAM J. Control Optim., issue dedicated to W.H. Fleming, 31, 439-469

5. Bouchard B. (2000). Stochastic target problem with jump-diffusion process. Stochastic Processes Appl. (forthcoming)

6. Bertsekas D.P., Shreve S.E. (1978). Stochastic optimal control: the discrete time case. Mathematics in Science and Engineering 139. New York: Academic Press

7. Broadie M., Cvitanić J., Soner H.M. (1998). Optimal replication of contingent claims under portfolio constraints. The Rev. Financial Stud. 11, 59-79

8. Chen Y.-G., Giga Y., Goto S. (1991). Uniqueness and existence of viscosity solutions of generalized mean curvature flow equations. J. Differential Geom. 33, 749-786

9. Crandall M., Ishii H., Lions P.-L. (1992). User's guide to viscosity solutions of second order partial differential equations. Bulletin AMS 27, 1-67

10. Cvitanić J., Ma J. (1996). Hedging options for a large investor and forward-backward SDE's. Ann. Appl. Probab. 6, 370-398

11. Cvitanić J., Pham H., Touzi N. (2000). Super-replication in stochastic volatility models under portfolio constraints. J. Appl. Probab. 36, 523-545

12. Doob J.L. (1994). Measure Theory. Berlin Heidelberg New York: Springer

13. Evans L.C., Spruck J. (1991). Motion of level sets by mean curvature. J. Differential Geom. 33, 635-681

14. Föllmer H., Kramkov D. (1997). Optional decomposition under constraints. Probab. Theory Related Fields 109, 1-25

15. Fleming W.H., Soner H.M. (1993). Controlled Markov Processes and Viscosity Solutions. New York, Heidelberg, Berlin: Springer

16. Gihman I.I., Skorokhod A.V. (1972). Stochastic Differential Equations. New York, Heidelberg, Berlin: Springer

17. Kabanov Yu. (1999). Hedging and liquidation under transaction costs in currency markets. Finance and Stochastics 3 (2), 237-248

18. Karatzas I., Shreve S. (1998). Methods of Mathematical Finance. New York, Heidelberg, Berlin: Springer

19. Gage M., Hamilton R. (1986). The heat equation shrinking convex plane curves. J. Differential Geom. 23, 69-95

20. Grayson M.A. (1987). The heat equation shrinks embedded plane curves to round points. J. Differential Geom. 26, 285-314

21. Huisken G. (1984). Flow by mean curvature of convex surfaces into spheres. J. Differential Geom.. 20, 237-266

22. Pardoux E., Peng S (1992). Backward stochastic differential equations and quasilinear parabolic partial differential equations. Stochastic partial differential equations and their applications (Charlotte, NC, 1991), Lecture Notes in Control and Inform. Sci. 176, Berlin: Springer 200-217

23. Peng S. (1991). Probabilistic interpretation for systems of quasilinear parabolic partial differential equations. Stochastics Stochastics Rep. 37, 61-74

24. Revuz D., Yor M. (1999). Continuous Martingales and Brownian Motion, 3rd edition. New York, Berlin: Springer

25. Soner H.M. (1993). Motion of a set by the curvature of its boundary. J. Differential Equations 101, 313-372

26. Soner H.M., Touzi N. (2000). Super-replication under Gamma constraints. SIAM J. Control Optim. 39, 73-96

27. Soner H.M., Touzi N. (2001). Stochastic target problems, dynamic programming, and viscosity solutions. SIAM J. Control Optim. (forthcoming)

28. Soner H.M., Touzi N. (2001). Stochastic representation of mean curvature type geometric flows (Preprint)

29. Soner H.M., Touzi N. (2001). A stochastic representation for the level set equations (Preprint)

30. Touzi N. (1998). Direct characterization of the value of super-replication under stochastic volatility and portfolio constraints. Stochastic Processes and their Applications (forthcoming) 